\title{
Genes influence the amplitude and timing of brain hemodynamic responses
}

Zuyao Y. Shan ${ }^{1}$, Anna A. E. Vinkhuyzen ${ }^{2}$, Paul M. Thompson ${ }^{3}$, Katie L. McMahon ${ }^{1}$, Gabriëlla A. M. Blokland ${ }^{1,4}$, Greig I. de Zubicaray ${ }^{5}$, Vince Calhoun ${ }^{6}$, Nicholas G. Martin ${ }^{4}$, Peter M. Visscher ${ }^{2}$, Margaret J. Wright ${ }^{4}$, David C. Reutens ${ }^{1}$

${ }^{1}$ Centre for Advanced Imaging, The University of Queensland, Brisbane, QLD 4072, Australia; ${ }^{2}$ Queensland Brain Institute, The University of Queensland, Brisbane, QLD 4072, Australia; ${ }^{3}$ Imaging Genetics Center, Institute of Neuroimaging and Informatics, Keck School of Medicine of University of Southern California, Marina del Rey, CA 90292, United States; ${ }^{4}$ QIMR Berghofer Medical Research Institute, Brisbane, QLD 4029, Australia; ${ }^{5}$ School of Psychology, The University of Queensland, Brisbane, QLD 4072, Australia; ${ }^{6}$ The Mind Research Network, Department of Electrical and Computer Engineering, The University of New Mexico, Albuquerque, NM 87313, United States.

Correspondence: David C. Reutens, MD; Centre for Advanced Imaging; The University of Queensland; St Lucia, Brisbane, QLD 4072, Australia; T: +61 (7) 336 4237, F: +61 (7) 33653833; E: $\underline{\text { d.reutens@uq.edu.au }}$

Abbreviation title: Heritability of brain hemodynamic responses 


\begin{abstract}
In functional magnetic resonance imaging (fMRI), the hemodynamic response function (HRF) reflects regulation of regional cerebral blood flow in response to neuronal activation. The HRF varies significantly between individuals. This study investigated the genetic contribution to individual variation in HRF using fMRI data from 125 monozygotic (MZ) and 149 dizygotic (DZ) twin pairs. The resemblance in amplitude, latency, and duration of the HRF in six regions in the frontal and parietal lobes was compared between MZ and DZ twin pairs. Heritability was estimated using an ACE (Additive genetic, Common environmental, and unique Environmental factors) model. The genetic influence on the temporal profile and amplitude of HRF was moderate to strong (24\%-51\%). The HRF may be used in the genetic analysis of diseases with a cerebrovascular etiology.
\end{abstract}

Keywords: hemodynamic response function (HRF); neurovascular coupling; heritability; twin study. 


\section{Introduction}

The brain has limited energy reserves. As a consequence, its normal function is critically dependent on neurovascular coupling - the matching of blood flow with neuronal activity spatially and temporally (Iadecola, 2004). The signal change detected in functional magnetic resonance imaging (fMRI) reflects changes in blood oxygenation induced by neuronal activation. The temporal evolution of this signal change, termed the hemodynamic response function (HRF) reflects regulation of regional cerebral blood flow in response to neuronal activation. The shape of the HRF can be characterized by its height $(H)$ or maximum signal change, time to maximum signal change $(T)$, and full width at half maximum signal change $(W)$. These HRF characteristics are influenced by vasodilatory signaling, blood vessel stiffness, neurovascular coupling delay, venous transit time, and the time constant of autoregulatory feedback (Buxton et al., 2004; Stephan et al., 2007).

The HRF varies between individuals (Aguirre et al., 1998) with moderate reliability over time within an individual(Shan et al., 2014). Knowing how much of the individual variation is due to inherited or acquired factors may yield a better understanding of cerebrovascular function in health and disease. To assess the contribution of genetic factors to HRF variability, here we estimate the heritability of HRF characteristics for the first time.

We tested whether HRF characteristics are heritable using fMRI data acquired during an N-back working memory task in 125 monozygotic (MZ) and 149 dizygotic (DZ) twin pairs. The 0- and 2-back working memory task (Blokland et al., 2011) activate the left (L) and right $(\mathrm{R})$ middle frontal gyrus (MFG); $\mathrm{L}$ and $\mathrm{R}$ supramarginal gyrus (SMG); and $\mathrm{L}$ and $\mathrm{R}$ angular gyrus (AG). We estimated the HRF in each of these regions by fitting the convolution of the HRF, modeled as the sum of 2 gamma functions with 6 parameters, and a function representing neuronal activity, to the time course of the fMRI signal. We selected 
this HRF model because of its performance in terms of precision, accuracy and parameter identifiability (Shan et al., 2014).

\section{Materials and Methods}

fMRI data. Data were acquired as part of a prior fMRI study (Blokland et al., 2011) forming part of the Queensland Twin Imaging Study (QTIMS) (de Zubicaray et al., 2008). The retrospective use of the data was approved by the Human Research Ethics Committee of the QIMR Berghofer Medical Research Institute and The University of Queensland in compliance with the Australia National Statement on Ethical Conduct in Human Research.

This study retrieved data from 125 monozygotic (MZ) and 149 dizygotic (DZ) twin pairs consisting of 340 females and 208 males with a mean age of $22.43 \pm 2.47$ SD (age range, $18-29$ years). The detailed demographic data of the twin sample was reported in the previous study (Blokland et al., 2011). There are 92 and 57 DZ twin pairs with same and different genders respectively. The average full-scale intelligence quotient (FIQ) of the participants was slightly higher than that of the general population, but still followed a normal distribution (Blokland et al., 2011). There was no significant difference between cotwins of either zygosity in gestational age, birth weight, or parental socioeconomic status.

Participants performed the 0 - and 2-back versions of the N-back working memory task. The detailed fMRI experimental procedure is described in detail in previous reports (Blokland et al., 2008; Callicott et al., 1998). In the N-back task, a series of numbers are presented on a screen. The 0-back condition required participants to respond to the number currently shown on the screen. The 2-back condition required participants to respond to the number presented 2 trials earlier. The number was presented for $200 \mathrm{~ms}$ with an $800 \mathrm{~ms}$ interval between stimuli, with 16 trials per block. In total, 16 alternating blocks were performed for the two conditions continuously ( 8 blocks per condition). 
The 3D T1-weighted MR image and echo planar imaging (EPI) data analyzed in this study were acquired on a 4T Bruker Medspec whole body scanner (Bruker, Germany). The 3D T1-weighted images were acquired using a MP-RAGE pulse sequence $(\mathrm{TR}=2500 \mathrm{~ms}$, $\mathrm{TE}=3.83 \mathrm{~ms}, \mathrm{~T} 1=1500 \mathrm{~ms}$, flip angle $=15$ degrees, $0.89 \times 0.89 \times 0.89 \mathrm{~mm}^{3}$ ). For each participant, 127 EPI data sets $(\mathrm{TR}=2.1 \mathrm{~s}, \mathrm{TE}=30 \mathrm{~ms}$, flip angle $=90$ degrees, $3.6 \times 3.6 \times$ $3.0 \mathrm{~mm}^{3}$ ) were acquired continuously during the tasks.

Task performance during scanning was recorded and summarized by accuracy and response time. Task accuracy was defined as the ratio between the number of correct 2-back trials to the total number of 2-back trials. Task response time (RT) was defined as the averaged time between stimulus onset and subject response across all 2-back trials.

Genotyping. Details regarding the zygosity determination are as described previously (Wright and Martin, 2004). Zygosity was confirmed by genome-wide single nucleotide polymorphism genotyping (Illumina $610 \mathrm{~K}$ chip).

Image analysis. fMRI data were analyzed using Statistical Parametric Mapping (SPM8, the Wellcome Trust Centre for Neuroimaging, London, UK). The first five EPI volumes were discarded to ensure that tissue magnetization had reached steady state. The spatial preprocessing included two pass motion correction (Friston et al., 1995) and spatial normalization to the average brain T1 template (Ashburner and Friston, 1999). Normalized volumes were smoothed with an $8 \times 8 \times 8 \mathrm{~mm}^{3}$ full width, half maximum Gaussian kernel. We first determined locations of activation using the general linear model with a finite impulse response (FIR) basis function. The FIR was chosen as the basis function to minimize the assumptions made about the form of the hemodynamic response function (HRF). The 2back minus 0-back t-contrast images were entered into a group-level random-effect (RFX) one-sample t-test to identify the common activation voxels $(\alpha<0.05$ with family wise error rate adjustment for multiple comparisons). The anatomical structures containing common 
activation voxels were selected as anatomic structures for HRF modeling: left (L-) and right (R-) middle frontal gyrus (MFG); L- and R- supramarginal gyrus (SMG); and L- and Rangular gyrus (AG) (Fig. 1c). These structures have been robustly associated with working memory in previous studies (Olesen et al., 2004). Regions of interest (ROIs) were defined as the overlap of an existing probabilistic atlas of each structure in stereotaxic coordinate space (Shattuck et al., 2008) with group activation regions. For each participant, the fMRI time course was extracted by averaging the signal intensity at each time point in the voxels with the top $12.5 \%$ of SPM t statistics within each ROI.

Hemodynamic response function (HRF) modeling. The detailed methodology of HRF modeling has previously been reported in Shan et al. (Shan et al., 2014). The HRF was estimated by fitting the fMRI time course with the convolution of HRF and neuronal activity functions. ROIs without fMRI activation (i.e. peak change in $\mathrm{HRF}<0.2 \%$ ) were excluded pairwise from heritability analysis. The number of $M Z$ and $D Z$ pairs with fMRI activation in each ROI are summarized in Table 1. The modeled HRFs were summarized by three characteristics: height $(H)$, the maximum percent signal change; time-to-peak $(T)$, the time for the signal to reach its maximum value; and width $(W)$ as full width at the half maximum (Fig. 1b). These characteristics reflect the maximum $(H)$, latency $(T)$, and duration $(W)$ of blood oxygenation changes caused by changes in local blood flow coupled to local neuronal activity.

Two neuronal activity functions were convolved with the HRF to fit the fMRI time course.

(1) The fixed neuronal activity function assumes that the neuronal activity is the same as the time course of the stimuli presented in the experiments. It was constructed with a fixed start time specified by the experimental design and uniform amplitude for each trial within all blocks (Fig. 1b). (2) The flexible neuronal activity function allows variation in the delay in the neuronal response and in the response amplitude for each trial in a block of tasks: 


$$
u(t+)=\left\{\begin{array}{cc}
{ }_{i} / m, & t=\text { stimulus on time } \\
0 & t=\text { others }
\end{array}\right.
$$

in which $t$ is the actual time from the start of task, $\tau$ is delay time, $\beta_{i}$ are amplitude parameters $(i=1,2, \ldots 16$ in this study), and $m$ is the number of trials in each block ( $m=16$ in this study) (Fig. 1e). The delay was initially set to 0 and all amplitude parameters were set to 1. Non-linear least squares fitting was then used to estimate the delay time and amplitude parameters for neuronal activity changes that yielded the best fit for each individual. Further, the median amplitude, the interquartile range (IQR) of amplitude and the approximate entropy (ApEn) of neuronal activity changes were calculated. ApEn quantifies the unpredictability of fluctuations in a time series, i.e. ApEn is small for predictable time series with repetitive patterns (Pincus, 1991). ApEn was calculated as:

$$
\operatorname{ApEn}(S, m, r)=\ln \left[\frac{C_{m}(r)}{C_{m+1}(r)}\right], C_{m}(r)=\frac{{ }_{i=1}^{N+1} C_{i m}(r)}{N \quad m+1}, \text { and } C_{i m}(r)=\frac{n_{i m}(r)}{N \quad m+1}
$$

where $S$ represents neuronal activity changes, $m$ is the pattern length ( $m=2$ in this study), $r$ is the similarity criterion ( $r=2 \times$ standard deviation of $S$ in this study), $N$ is the length of $S$, $n_{i m}(r)$ is the number of patterns that are similar to pattern $i$ determined by the similarity criterion $r$, and $C_{i m}(r)$ is the fraction of patterns of length $m$ that resemble the pattern of the same length to pattern $i$.

Simulation of HRF modeling with flexible neuronal activity function. Simulation studies were performed to test the ability to model neuronal responses using a flexible neuronal activity function. The fMRI data were simulated by the convolution of the HRF and the neuronal activity function (Shan et al., 2014). The Balloon Model was used to generate the HRF. To examine the ability to estimate variations in the amplitude of neuronal activity changes, this was decreased in 5\% increments between $10 \%$ and $90 \%$ of activity. The number 
of trials in each block affected by the change in amplitude was varied systematically from the first trial in each block to the first 15 of a total of 16 trials in each block. The neuronal activity function was convolved with the simulated HRF and sampled to generate a simulated fMRI time course. Estimation of HRF characteristics was performed as described above. $H$ and $T$ of HRFs modeled from the simulated fMRI time course were compared with the true values, calculated from the HRF that was used to generate simulated data.

We also tested the ability to estimate HRF characteristics in the presence of random variations in neuronal activity and of noise. To ensure that a realistic temporal signal to noise ratio (SNR) was used in the simulation, we first estimated the temporal SNR in the fMRI datasets. Cubic volumes of interest (VOI), $3 \times 3 \times 3$ voxels in size, were selected from the anterior cerebellum and the mid corpus callosum. Temporal SNRs were calculated as the ratio between the mean intensity in the fMRI time series (122 EPI volumes) and the standard deviation. The neuronal activity function was randomly varied and convolved with the HRF. Random noise was then added to the generated time course, with a temporal SNR of 150 , selected on the basis of the histogram of estimated SNR from the whole group. The simulated fMRI time course was sampled with a TR of 2.1s. The HRFs were then estimated using fixed and flexible neuronal activity functions. The simulation was executed 100 times. The number of simulation execution was determined by obtaining stable variability and performance (Shan et al., 2014). $H$ and $T$ of HRFs modeled from the simulated fMRI time course were compared with the true values, calculated from the HRF that was used to generate simulated data. The estimation error for the neuronal activity function was evaluated by the relative error in the time to onset of neuronal activity and by relative error in the amplitude of neuronal activity in all trials.

Correlations between estimated HRF characteristics, neuronal activation, and performance data. Pearson correlations between task performance and HRF characteristics 
or neuronal activity changes were calculated using SPSS20 (IBM, New York). Bivariate tests of significant difference in correlation coefficient from zero with two tails followed with false discovery rate (FDR) correction (FDR-q < 0.05) (Storey, 2002) was used to determine statistical significance.

Heritability analysis of HRF characteristics. To examine the heritability of the $\mathrm{HRF}$, we first compared the resemblance of HRF characteristics in MZ and DZ twin pairs. For each HRF characteristic, the intra-class correlations (ICC) measuring absolute agreement in HRF characteristics within MZ and DZ twin pairs were determined with a two way random effects model using SPSS20 (IBM, New York). Tests of significant difference in ICC from zero was followed with Bonferroni correction $(P<0.0028)$ for multiple comparisons of $18 \mathrm{HRF}$ characteristics to determine statistical significance.

The normality of all HRF characteristics was confirmed using Q-Q plots in SPSS20 (IBM, New York). The relative contribution of additive genetic factors to the variance of each HRF characteristic was estimated using a structural equation model implemented in the software package Mx (Neale and Miller, 1997). Phenotypic variances of MZ and DZ twins were constrained to be equal. Age and gender were included as covariates. MZ twin pairs were expected to share all of their genetic material whereas DZ twin pairs were expected to share, on average, half of their genetic material. Variances of HRF characteristics were decomposed into additive genetic factors (A), common environmental factors $(\mathrm{C})$, and unique environmental factors, which also included measurement errors (E). For the full ACE model, common environmental factors were estimated to be zero. Thus, the significance of genetic factors was determined by comparing the goodness-of-fit of the AE model and the E model. Tests of significant difference in goodness-of-fit from zero were followed with Bonferroni correction $(p<0.0028)$ for multiple comparisons of $18 \mathrm{HRF}$ characteristics. We also modeled 
non-additive genetic (D) influences using an ADE model by introducing an expected coefficient of 0.25 for non-additive effect. 


\section{Results}

Heritability of HRF characteristics modeled with fixed neuronal activity function. MZ twins showed significant resemblance in all HRF characteristics in all regions of interest (ROIs) except $W$ in right supramarginal gyrus, while DZ twins did not (Table 2). The high ICCs and the significant resemblance within MZ pairs and significant ICC difference between MZ and DZ pairs are strong evidence that HRF characteristics are heritable.

Of all models tested, the AE model had the lowest Akaike Information Criterion (AIC) value for all HRF parameters and regions except for $W$ in the left middle frontal gyrus (Table 3). There were significant $(P<0.0028)$ genetic contributions to $H$ and $T$ for most ROIs (Fig. 1a). These results indicate a significant genetic influence on the amplitude and latency of the hemodynamic response induced by the activation of brain regions involved in working memory. The full ACE model estimated C to be zero. The ADE model goodness-offit was slightly better (lower AIC) than for the ACE model but inferior to that of the AE model for $H$ and $T$ in all regions except for $T$ in right middle frontal gyrus. Model goodnessof-fit did not change significantly when $\mathrm{D}$ was omitted from the model (ADE vs. AE). However, the goodness-of-fit decreased with omission both A and D, further confirming a genetic influence (additive and/or dominant) on the amplitude and latency of the hemodynamic response (Table 3).

Simulation of HRF modeling with flexible neuronal activity function. Estimation errors of HRF characteristics without noise are summarized in pseudocolor maps (Fig. 2). Use of a fixed neuronal activity function results in estimation errors for $H$ if the actual neuronal activity does not follow the fixed function whereas errors in the estimation of $T$ are not seen if the change in the amplitude of actual neuronal activity is less than 50\%. HRF modeling with a flexible neuronal activity function improved the accuracy of HRF estimation when neuronal activity varied. 
With random noise (temporal SNR $=150$ ), $H$ and $T$ were estimated more accurately with a flexible neuronal activity function than with a fixed neuronal activity function (Fig. 3). As expected, the flexible neuronal activity function estimated the time course of neuronal activity more accurately than the fixed neuronal activity function (Fig. 4).

Correlations between estimated HRF characteristics, neuronal activation, and performance data. Several HRF characteristics were significantly correlated with task performance when a fixed neuronal activity function was used. HRF $H$ in the L-MFG and LAG was negatively correlated with performance accuracy, with both having a Pearson correlation coefficient $(\rho)$ equal to $-0.11($ FDR-q $<0.05$ and for all correlations reported below). The HRF $H$ was positively correlated with response time in L-MFG $(\rho=0.13)$, LSMG $(\rho=0.12)$, L-AG $(\rho=0.17)$, and R-AG $(\rho=0.13)$. No significant correlation was found between HRF characteristics and task performance when a flexible neuronal activity function was used.

There were no significant correlations between the estimated neuronal activity delays and performance data. Median of neuronal activity amplitude changes in L-MFG $(\rho=-0.07)$, R-MFG $(\rho=-0.06)$, L-SMG $(\rho=-0.09)$, and R-SMG $(\rho=-0.12)$ were negatively correlated with response accuracy. Median neuronal activity amplitude changes in L-MFG $(\rho=0.11)$ and L-AG $(\rho=0.07)$ were positively correlated with response time. The ApEns of neuronal activity estimated from the L-MFG $(\rho=-0.08)$ and R-AG $(\rho=-0.07)$ were negatively correlated with response accuracy. The ApEn of neuronal activity estimated from the L-MFG $(\rho=0.08)$ was positively correlated with response time.

Heritability of HRF characteristics modeled with flexible neuronal activity function. When the flexible neuronal activity function was used, the ICCs for $H$ and $T$ in MZ twin pairs were similar to those modeled with the fixed neuronal activity function (Table 2 ) and the findings from structural equation modeling showed a similar genetic influence on $H$ and $T$ 
(Fig. 1d and Table 4). The ICCs for median amplitude and approximate entropy of the estimated neuronal function in both $\mathrm{MZ}$ and $\mathrm{DZ}$ twin pairs were not significant and no significant genetic contribution was observed with structural equation modeling. 


\section{Discussion}

By comparing resemblance of HRF characteristics within $\mathrm{MZ}$ and DZ twin pairs, we have provided evidence for a significant genetic influence on the regulation of regional cerebral blood flow. The HRF was modeled by fitting the convolution of the HRF with the neuronal activity function to the fMRI time course. In most fMRI analyses, a fixed neuronal activity function, which assumes that the neuronal activity is the same as the time course of the stimuli presented in the experiments, is used. However, to test whether the observed heritability of HRF characteristics was due to the assumed time course of neuronal activity, we also used a flexible neuronal activity function to model the time course of the fMRI signal, fitting its convolution with the HRF to the fMRI time course to estimate both the HRF and neuronal activity. In simulation studies, the flexible neuronal activity function improves the accuracy of HRF estimation when the neuronal response to stimuli varies. Poorer performance (lower accuracy and longer mean response times) was associated with higher amplitude and more irregular neuronal activity. The relationship between HRF characteristics and performance differed between flexible and fixed neuronal activity functions in a manner consistent with the former being a more accurate representation of neuronal activity. This suggests that inaccurate representation of neuronal activity affects the estimation of HRF characteristics. In contrast, estimated HRF characteristics did not correlate with performance when the flexible neuronal activity function was used. The median amplitude change and approximate entropy, a measure of the temporal regularity of a signal (Pincus, 1991), of the neuronal activity function correlated with in-scanner subject performance providing support for the flexible neuronal activity function as an accurate representation of neuronal activity. The genetic influence on HRF characteristics was similar for fixed and flexible neuronal activity functions. Hence we conclude that the observed heritability reflects genetic 
influences on regional blood flow regulation per se rather than an effect mediated via neuronal activity.

Based on our sensitivity analyses of fMRI signal physiological determinants with the generalized Balloon Model (Buxton et al., 2004; Stephan et al., 2007) (Supplementary Fig. S1 and Table S1), we believe that the heritability in HRF amplitude found here is most likely attributable to genes regulating vasodilator signaling or blood vessel stiffness. Candidate genes include those involved in synthesis of vasodilators such as nitric oxide synthase 1 (NOS1) and cyclooxygenase $(C O X)$. Indomethacin, a cyclooxygenase inhibitor, has been shown to decrease the blood oxygenation level-dependent response in fMRI (Bruhn et al., 2001). Furthermore, the amplitude of the HRF is affected by COX genotypes (Hahn et al., 2011). Heritability for vascular compliance has been demonstrated for the venous system in which heritability is about $90 \%$ (Brinsuk et al., 2004). Here, genes for vessel wall proteins such as desmuslin, an intermediate filament protein in the smooth muscle cell wall (Anwar et al., 2012), may be important genetic influences on blood vessel stiffness. We demonstrated a significant genetic contribution to individual variation in $T$ and have previously shown that $H$ and $T$ can be estimated accurately and independently (Shan et al., 2014). Our sensitivity analyses showed that $T$ is most strongly influenced by neurovascular coupling delay, reflecting the influence of genes involved in vascular signaling.

Significant resemblance in $W$ in $\mathrm{MZ}$ twins was observed for estimates made with a fixed but not a flexible neuronal activity function suggesting that some of the observed heritability in the former was due to similarity in the time course of neuronal activity within MZ twin pairs. $W$ is only moderately reproducible (Shan et al., 2014), imposing a ceiling on estimates of heritability. In light of these two observations, the present data do not allow us to conclude that there are significant genetic influences on $W$. 
In the current study, the HRF was estimated from block design fMRI data. Paradigm design was an unavoidable limitation of the retrospective analysis of fMRI data that had originally been collected to study the heritability of spatial patterns of functional activation with a working memory task (Blokland et al., 2011). We note that block designs are not optimal for HRF estimation and that random event-related designs and $m$-sequence designs have higher estimation efficiency (Maus et al., 2012). However, in our previous work, we demonstrated that HRF parameters can still be reliably estimated from block design fMRI data (Shan et al., 2014).

Our findings indicate that genetic factors contribute to individual variability in the coupling of the hemodynamic response to neuronal activity. The findings identify a new potential contributor to the heritability of some neurological diseases. The HRF may be a bridge between genes and diseases such as small vessel disease, a major cause of cognitive decline and gait disability with aging, in which altered neurovascular coupling has recently been implicated as playing a central pathobiological role (Gorelick et al., 2011; Iadecola, 2013; Zacchigna et al., 2008). A growing literature also implicates abnormal neurovascular mechanisms in the development and progression of Alzheimer's disease. Vascular insufficiency activates the cleavage of the amyloid precursor protein (Kitaguchi et al., 2009) and promotes amyloid formation (Okamoto et al., 2012) and tau phosphorylation (Koike et al., 2010) in Alzheimer's disease and preliminary evidence suggests that control of vascular risk factors may delay the progression of the disorder (Deschaintre et al., 2009; Richard et al., 2010). 
Acknowledgements: We thank the twins for participating in this study; research nurses Marlene Grace and Ann Eldridge at QIMR-Berghofer for twin recruitment; radiographers Matthew Meredith, Peter Hobden, and Aiman Al Najjar from the Centre for Advanced Imaging, The University of Queensland, for data acquisition; research assistants Kori Johnson and Lachlan Strike at the QIMR-Berghofer Medical Research Institute, for preparation and management of the imaging files; and Shona Osborne from the Centre for Advanced Imaging, The University of Queensland, for scientific editing. Data acquisition and analysis were supported by the Eunice Kennedy Shriver National Institute of Child Health \& Human Development (Grant RO1 HD050735) and National Health and Medical Research Council, Australia (Project Grants 496682, 1009064, and Program Grant 628952).

The authors declare no competing financial interests. 


\section{References}

Aguirre, G.K., Zarahn, E., D'Esposito, M., 1998. The variability of human, BOLD hemodynamic responses. Neuroimage 8, 360-369.

Anwar, M.A., Georgiadis, K.A., Shalhoub, J., Lim, C.S., Gohel, M.S., Davies, A.H., 2012. A Review of Familial, Genetic, and Congenital Aspects of Primary Varicose Vein Disease. Circulation-Cardiovascular Genetics 5, 460-466.

Ashburner, J., Friston, K.J., 1999. Nonlinear spatial normalization using basis functions. Human Brain Mapping 7, 254-266.

Blokland, G.A.M., McMahon, K.L., Hoffman, J., Zhu, G., Meredith, M., Martin, N.G., Thompson, P.M., de Zubicaray, G.I., Wright, M.J., 2008. Quantifying the heritability of taskrelated brain activation and performance during the N-back working memory task: A twin fMRI study. Biological Psychology 79, 70-79.

Blokland, G.A.M., McMahon, K.L., Thompson, P.M., Martin, N.G., de Zubicaray, G.I., Wright, M.J., 2011. Heritability of Working Memory Brain Activation. Journal of Neuroscience 31, 10882-10890.

Brinsuk, M., Tank, J., Luft, F.C., Busjahn, A., Jordan, J., 2004. Heritability of venous function in humans. Arteriosclerosis Thrombosis and Vascular Biology 24, 207-211.

Bruhn, H., Fransson, P., Frahm, J., 2001. Modulation of cerebral blood oxygenation by indomethacin: MRI at rest and functional brain activation. Journal of Magnetic Resonance Imaging 13, 325-334.

Buxton, R.B., Uludag, K., Dubowitz, D.J., Liu, T.T., 2004. Modeling the hemodynamic response to brain activation. Neuroimage 23, S220-S233.

Callicott, J.H., Ramsey, N.F., Tallent, K., Bertolino, A., Knable, M.B., Coppola, R., Goldberg, T., van Gelderen, P., Mattay, V.S., Frank, J.A., Moonen, C.T.W., Weinberger, D.R., 1998. Functional magnetic resonance imaging brain mapping in psychiatry: Methodological issues illustrated in a study of working memory in schizophrenia. Neuropsychopharmacology 18, 186-196.

de Zubicaray, G.I., Chiang, M.-C., McMahon, K.L., Shattuck, D.W., Toga, A.W., Martin, N.G., Wright, M.J., Thompson, P.M., 2008. Meeting the Challenges of Neuroimaging Genetics. Brain Imaging and Behavior 2, 258-263.

Deschaintre, Y., Richard, F., Leys, D., Pasquier, F., 2009. Treatment of vascular risk factors is associated with slower decline in Alzheimer disease. Neurology 73, 674-680.

Dominicus, A., Skrondal, A., Gjessing, H.K., Pedersen, N.L., Palmgren, J., 2006. Likelihood ratio tests in behavioral genetics: problems and solutions. Behav Genet 36, 331-340.

Friston, K.J., Frith, C.D., Turner, R., Frackowiak, R.S.J., 1995. CHARACTERIZING EVOKED HEMODYNAMICS WITH FMRI. Neuroimage 2, 157-165.

Gorelick, P.B., Scuteri, A., Black, S.E., Decarli, C., Greenberg, S.M., Iadecola, C., Launer, L.J., Laurent, S., Lopez, O.L., Nyenhuis, D., Petersen, R.C., Schneider, J.A., Tzourio, C., 
Arnett, D.K., Bennett, D.A., Chui, H.C., Higashida, R.T., Lindquist, R., Nilsson, P.M., Roman, G.C., Sellke, F.W., Seshadri, S., 2011. Vascular contributions to cognitive impairment and dementia: a statement for healthcare professionals from the american heart association/american stroke association. Stroke 42, 2672-2713.

Hahn, T., Heinzel, S., Plichta, M.M., Reif, A., Lesch, K.P., Fallgatter, A.J., 2011. Neurovascular Coupling in the Human Visual Cortex Is Modulated by Cyclooxygenase-1 (COX-1) Gene Variant. Cerebral Cortex 21, 1659-1666.

Iadecola, C., 2004. Neurovascular regulation in the normal brain and in Alzheimer's disease. Nature Reviews Neuroscience 5, 347-360.

Iadecola, C., 2013. The pathobiology of vascular dementia. Neuron 80, 844-866.

Kitaguchi, H., Tomimoto, H., Ihara, M., Shibata, M., Uemura, K., Kalaria, R.N., Kihara, T., Asada-Utsugi, M., Kinoshita, A., Takahashi, R., 2009. Chronic cerebral hypoperfusion accelerates amyloid beta deposition in APPSwInd transgenic mice. Brain Res 1294, 202-210.

Koike, M.A., Green, K.N., Blurton-Jones, M., Laferla, F.M., 2010. Oligemic hypoperfusion differentially affects tau and amyloid-\{beta\}. Am J Pathol 177, 300-310.

Maus, B., van Breukelen, G.J.P., Goebel, R., Berger, M.P.F., 2012. Optimal design for nonlinear estimation of the hemodynamic response function. Human Brain Mapping 33, 1253-1267.

Neale, M.C., Miller, M.B., 1997. The use of likelihood-based confidence intervals in genetic models. Behavior Genetics 27, 113-120.

Okamoto, Y., Yamamoto, T., Kalaria, R.N., Senzaki, H., Maki, T., Hase, Y., Kitamura, A., Washida, K., Yamada, M., Ito, H., Tomimoto, H., Takahashi, R., Ihara, M., 2012. Cerebral hypoperfusion accelerates cerebral amyloid angiopathy and promotes cortical microinfarcts. Acta Neuropathol 123, 381-394.

Olesen, P.J., Westerberg, H., Klingberg, T., 2004. Increased prefrontal and parietal activity after training of working memory. Nature Neuroscience 7, 75-79.

Pincus, S.M., 1991. APPROXIMATE ENTROPY AS A MEASURE OF SYSTEMCOMPLEXITY. Proceedings of the National Academy of Sciences of the United States of America 88, 2297-2301.

Richard, E., Gouw, A.A., Scheltens, P., van Gool, W.A., 2010. Vascular care in patients with Alzheimer disease with cerebrovascular lesions slows progression of white matter lesions on MRI: the evaluation of vascular care in Alzheimer's disease (EVA) study. Stroke 41, 554556.

Shan, Z.Y., Wright, M.J., Thompson, P.M., McMahon, K.L., Blokland, G.G., de Zubicaray, G.I., Martin, N.G., Vinkhuyzen, A.A., Reutens, D.C., 2014. Modeling of the hemodynamic responses in block design fMRI studies. J Cereb Blood Flow Metab 34, 316-324.

Shattuck, D.W., Mirza, M., Adisetiyo, V., Hojatkashani, C., Salamon, G., Narr, K.L., Poldrack, R.A., Bilder, R.M., Toga, A.W., 2008. Construction of a 3D probabilistic atlas of human cortical structures. Neuroimage 39, 1064-1080. 
Stephan, K.E., Weiskopf, N., Drysdale, P.M., Robinson, P.A., Friston, K.J., 2007. Comparing hemodynamic models with DCM. Neuroimage 38, 387-401.

Storey, J.D., 2002. A direct approach to false discovery rates. Journal of the Royal Statistical Society Series B-Statistical Methodology 64, 479-498.

Wright, M.J., Martin, N.G., 2004. Brisbane adolescent twin study: Outline of study methods and research projects. Australian Journal of Psychology 56, 65-78.

Zacchigna, S., Lambrechts, D., Carmeliet, P., 2008. Neurovascular signalling defects in neurodegeneration. Nat Rev Neurosci 9, 169-181. 


\section{Figure Legends}

Fig. 1. Estimating hemodynamic response functions (HRFs) and their heritability. Heritability was estimated using a full ACE (Additive genetic, Common environmental, and unique Environmental factors) model. The ACE model estimated the common environmental factors to be zero except for $H(17 \%)$ and $W(18 \%)$ in the left angular gyrus when the HRFs were estimated with a flexible neuronal activity function. (a) Heritability of HRF characteristics estimated with a fixed neuronal activity function. (b) HRF estimation by fitting convolution of the HRF with a fixed neuronal activity function, corresponding to the time course of stimulus presentation, to the time course of fMRI signal. (c) Anatomic structures within which HRFs were estimated. Left (L-) and right (R-) middle frontal gyrus (MFG), angular gyrus (AG), and supramarginal gyrus (SMG) were activated by the working memory task. (d) Heritability of HRF characteristics estimated with a flexible neuronal activity function (e) HRF estimation by fitting convolution of the HRF with a flexible neuronal activity function to the time course of fMRI signal.

Fig. 2. Pseudocolor plots of the estimation errors of HRF characteristics $H(a, b)$ and $T(\mathrm{c}, \mathrm{d})$. The amplitude of the neuronal activity function was decreased in 5\% increments between $10 \%$ and $90 \%$ of activity (represented by the horizontal axis). The number of trials in each block affected by the change in amplitude was varied from the first trial in each block to the first 15 of a total of 16 trials in each block (represented by the vertical axis). The absolute differences between the estimated HRF characteristics and the true values were color-coded: the color bars represent estimation errors in $H$ between 0 and 1.2\% (a and b) and in $T$ between 0 s and 3.4s (c and d). (a) and (c) respectively represent the estimation errors of HRF $H$ and $T$ using the fixed neuronal activity function. (b) and (d) respectively represent the estimation errors of HRF $H$ and $T$ using the flexible neuronal activity function. Overall, HRF $H$ and $T$ are estimated more accurately when a flexible as opposed to a fixed neuronal activity 
function is used. While HRF modeling with a flexible neuronal activity function improved the accuracy of HRF estimation when neuronal activity varied, errors in the estimation of $T$ are not seen if the change in the amplitude of actual neuronal activity is less than $50 \%$.

Fig. 3. Box-and-whisker plot comparing the relative deviations of estimated HRF characteristics from the true values when flexible versus fixed neuronal activity functions were used. Outliers (red cross) were defined as exceeding 3 times the standard deviation; all data, including outliers without Winsorization, were considered in the analysis. The HRF characteristics estimated with the flexible neuronal activity function are more accurate (less deviation from the true values and smaller interquartile range) than those estimated using a fixed neuronal activity function.

Fig. 4. Box-and-whisker plot of deviations of estimated neuronal activity from true values in the simulation study. Outliers (red cross) were defined as exceeding 3 times the standard deviation; all data, including outliers without Winsorization, were considered in the analysis. (a) The relative deviations of estimated delays in onset of neuronal activity from true values. (b) The sum of relative errors in estimated amplitude of neuronal activity. The delay and amplitude of neuronal activity estimated with the flexible neuronal activity function are more accurate than those estimated using a fixed neuronal activity function. 
Table 1

Number of pairs with estimated HRFs for each region of interest (ROI)

ROI Fixed neuronal activity function Flexible neuronal activity function
MZ pairs
DZ pairs
MZ pairs
DZ pairs

L-MFG

94

124

94

116

L-SMG $\quad 5$

61

49

64

L-AG

68

93

71

83

R-MFG 95

133

89

131

R-SMG

64

85

67

90

R-AG

69

104

82

97

§: HRFs: hemodynamic response function characteristics, L-: left; R-: right; MFG: middle frontal gyrus; SMG: supramarginal gyrus; AG: angular gyrus; MZ: monozygotic twin; DZ: dizygotic twin 
Table 2

The intra-class correlations in HRF characteristics in twin pairs ${ }^{\S}$

Fixed neuronal activity functions $\quad$ Flexible neuronal functions

HRFs

MZ pairs (95\% CI) DZ pairs (95\% CI) $\mathrm{MZ}$ pairs (95\% CI) DZ pairs $(95 \% \mathrm{CI})$

\begin{tabular}{|c|c|c|c|c|}
\hline L-MFG- $H$ & $0.49 * *(0.23,0.66)$ & $0.23(0,0.46)$ & $0.52 * *(0.26,0.69)$ & $0.33 *(0.04,0.54)$ \\
\hline L-SMG- $H$ & $0.49 * *(0.12,0.71)$ & $0.14(0,0.49)$ & $0.5 * *(0.11,0.72)$ & $0.19(0,0.51)$ \\
\hline L-AG- $H$ & $0.56 * *(0.29,0.73)$ & $0.2(0,0.47)$ & $0.66 * *(0.46,0.79)$ & $0.49 *(0.19,0.68)$ \\
\hline R-MFG- $H$ & $0.56 * *(0.33,0.71)$ & $0.03(0,0.31)$ & $0.51 * *(0.26,0.68)$ & $0.36 *(0.11,0.54)$ \\
\hline R-SMG- $H$ & $0.59 * *(0.32,0.75)$ & $0.07(0,0.4)$ & $0.5 * *(0.18,0.69)$ & $0.17(0,0.46)$ \\
\hline R-AG- $H$ & $0.64 * *(0.41,0.78)$ & $0.09(0,0.38)$ & $0.47 * *(0.18,0.69)$ & $0.07(0,0.4)$ \\
\hline L-MFG- $T$ & $0.47 * *(0.2,0.65)$ & $0.26(0,0.48)$ & $0.54 * *(0.29,0.7)$ & $0.37 *(0.08,0.56)$ \\
\hline L-SMG- $T$ & $0.48 *(0.1,0.71)$ & $0.31(0,0.58)$ & $0.49 * *(0.11,0.71)$ & $0.03(0,0.42)$ \\
\hline $\mathrm{L}-\mathrm{AG}-T$ & $0.55 * *(0.27,0.72)$ & $0.08(0,0.39)$ & $0.51 * *(0.21,0.69)$ & $0.18(0,0.49)$ \\
\hline R-MFG- $T$ & $0.51 * *(0.27,0.68)$ & $0.01(0,0.3)$ & $0.55 * *(0.31,0.7)$ & $0.15(0,0.39)$ \\
\hline R-SMG- $T$ & $0.54 * *(0.24,0.72)$ & $0.28(0,0.53)$ & $0.49 * *(0.17,0.68)$ & $0.03(0,0.36)$ \\
\hline $\mathrm{R}-\mathrm{AG}-T$ & $0.51 * *(0.2,0.7)$ & $0.05(0,0.36)$ & $0.5^{* *}(0.22,0.68)$ & $0.13(0.34,0.43)$ \\
\hline L-MFG- $W$ & $0.4 * *(0.1,0.6)$ & $0.05(0.35,0.34)$ & $0.49 * *(0.21,0.67)$ & $0.2(0,0.45)$ \\
\hline L-SMG- $W$ & $0.51 * *(0.14,0.72)$ & $0.1(0,0.46)$ & $0.09(0,0.49)$ & $0.02(0,0.38)$ \\
\hline L-AG- $W$ & $0.53 * *(0.24,0.71)$ & $0.20(0,0.47)$ & $0.16(0,0.48)$ & $0.4^{*}(0.04,0.63)$ \\
\hline
\end{tabular}




$\begin{array}{ccccc}\text { R-MFG- } W & \mathbf{0 . 4 8} * *(\mathbf{0 . 2 2}, \mathbf{0 . 6 5}) & 0.15(0,0.39) & \mathbf{0 . 3 4} * \mathbf{( 0 , 0 . 5 7 )} & 0.2(0,0.43) \\ \text { R-SMG- } W & 0.34(0,0.6) & 0.17(0,0.46) & 0.19(0,0.51) & 0.03(0,0.43) \\ & & & & \\ \text { R-AG- } W & \mathbf{0 . 3 9} *(\mathbf{0 . 0 2}, \mathbf{0 . 6 2}) & 0.03(0,0.34) & \mathbf{0 . 5 2} * *(\mathbf{0 . 2 5}, \mathbf{0 . 6 9}) & 0.04(0,0.42)\end{array}$

§: Intra-class correlations were calculated using a two way random model and the type of absolute agreement. There was no significant effect of age and sex on HRF characteristics. HRFs: hemodynamic response function characteristics, L-: left; R-: right; MFG: middle frontal gyrus; SMG: supramarginal gyrus; AG: angular gyrus; MZ: monozygotic twin; DZ: dizygotic twin; Given that ICC cannot be negative, the negative ICC values rendered by SPSS were set to zeros; **: significant correlations with Bonferroni corrections $(P<0.0028)$; *: moderate correlations $(P<0.05)$. 


\section{Table 3}

Statistical modeling of hemodynamic response function characteristics estimated with a fixed neuronal activity function§

\begin{tabular}{|c|c|c|c|c|c|c|c|c|c|c|c|c|c|c|c|c|c|c|c|}
\hline \multirow{3}{*}{ HRFs } & \multicolumn{10}{|c|}{ Model fit } & \multicolumn{9}{|c|}{ Model comparison } \\
\hline & \multicolumn{2}{|c|}{ ACE } & \multicolumn{2}{|c|}{$\mathrm{ADE}$} & \multicolumn{2}{|c|}{$\mathrm{CE}$} & \multicolumn{2}{|c|}{$\mathrm{AE}$} & \multicolumn{2}{|c|}{$\mathrm{E}$} & \multicolumn{2}{|c|}{ ACE vs. AE } & \multicolumn{2}{|c|}{ ACE vs. CE } & \multicolumn{2}{|c|}{ ADE vs. AE } & \multicolumn{2}{|c|}{ ADE vs. E } & AE vs. E \\
\hline & $-2 \mathrm{LL}$ & AIC & $-2 \mathrm{LL}$ & AIC & $-2 \mathrm{LL}$ & AIC & $-2 L L$ & AIC & $-2 \mathrm{LL}$ & AIC & $\Delta \chi^{2}$ & $P$ & $\Delta \chi^{2}$ & $P$ & $\Delta \chi^{2}$ & $P$ & $\Delta \chi^{2}$ & $P$ & $\Delta \chi^{2}$ \\
\hline L-MFG- $H$ & 761.49 & -158.51 & 761.10 & -158.90 & 765.50 & -156.50 & 761.49 & -160.51 & 777.42 & -146.58 & 0.00 & 1 & 4.00 & 0.04 & 0.39 & 0.53 & 16.32 & 2.85E-04 & 15.93 6.57E-05 \\
\hline L-SMG- $H$ & 403.03 & -264.97 & 402.96 & -265.04 & 404.54 & -265.46 & 403.03 & -266.97 & 410.75 & -261.25 & 0.00 & 1 & 1.51 & 0.22 & 0.08 & 0.78 & 7.79 & 0.02 & 7.72 5.47E-03 \\
\hline L-AG- $H$ & 723.84 & -54.15 & 723.45 & -54.55 & 726.22 & -53.78 & 723.84 & -56.15 & 732.57 & -49.43 & 0.00 & 1 & 2.37 & 0.12 & 0.39 & 0.53 & 9.12 & 0.01 & 8.72 3.14E-03 \\
\hline R-MFG- $H$ & 713.04 & -238.96 & 709.69 & -242.31 & 718.71 & -235.29 & 713.03 & -240.96 & 724.26 & -231.74 & 0.00 & 1 & 5.67 & 0.02 & 3.34 & 0.07 & 14.57 & 6.87E-04 & 11.22 8.07E-04 \\
\hline R-SMG- $H$ & 461.48 & -316.52 & 460.44 & -317.56 & 464.88 & -315.12 & 461.48 & -318.52 & 472.30 & -309.70 & 0.00 & 1 & 3.40 & 0.06 & 1.04 & 0.31 & 11.86 & $2.66 \mathrm{E}-03$ & 10.82 1.00E-03 \\
\hline R-AG- $H$ & 505.15 & -330.85 & 502.03 & -333.97 & 511.60 & -326.40 & 505.15 & -332.85 & 518.96 & -321.04 & 0.00 & 1 & 6.45 & 0.01 & 3.12 & 0.08 & 16.93 & 2.11E-04 & 13.81 2.02E-04 \\
\hline L-MFG- $T$ & 1403.89 & 483.89 & 1403.88 & 483.88 & 1406.17 & 484.17 & 1403.89 & 482.89 & 1419.67 & 495.67 & 0.00 & 1 & 2.28 & 0.13 & $4.59 \mathrm{E}-03$ & 30.95 & 15.79 & 3.72E-04 & 15.79 7.09E-05 \\
\hline L-SMG-T & 1112.68 & 444.68 & 1111.78 & 443.78 & 1115.63 & 445.63 & 1112.68 & 442.68 & 1120.93 & 448.93 & 0.00 & 1 & 2.95 & 0.09 & 0.90 & 0.34 & 9.16 & 0.01 & 8.25 4.06E-03 \\
\hline
\end{tabular}




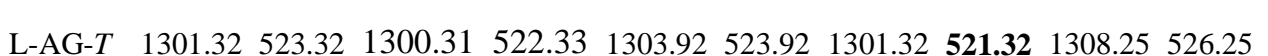

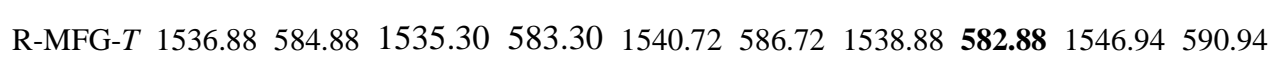

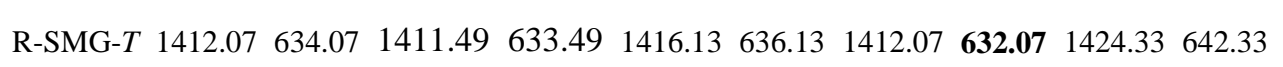

R-AG- $T \quad 1504.21 \quad 668.21 \quad 1500.99664 .99 \quad 1507.44 \quad 669.44 \quad 1504.21 \quad \mathbf{6 6 6 . 2 1} 1509.69669 .69$

L-MFG- $W \quad 1382.74 \quad 462.74 \quad 1382.34 \quad 462.34 \quad 1383.51 \quad 461.51 \quad 1382.74 \quad 460.74 \quad 1384.63 \quad \mathbf{4 6 0 . 6 3}$

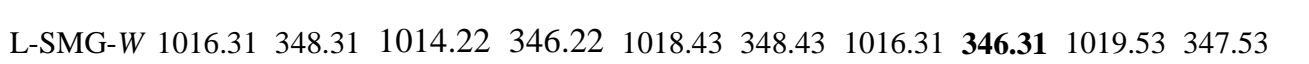

$$
\text { L-A }
$$

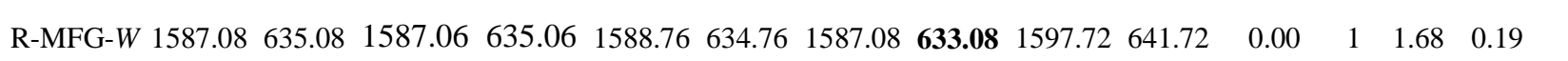

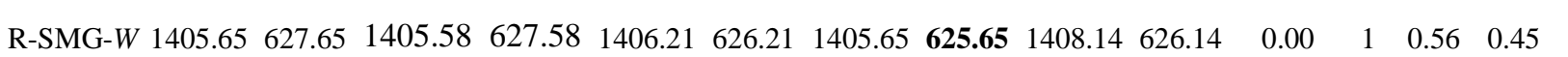

$\begin{array}{lllllllllllllllllllll}\text { R-AG- } W & 1520.72 & 684.72 & 1517.82 & 681.82 & 1522.93 & 684.93 & 1520.72 & \mathbf{6 8 2 . 7 2} & 1524.17 & 684.17 & 0.00 & 1 & 2.21 & 0.14 & 2.90 & 0.09 & 6.35 & \mathbf{0 . 0 4} & 3.45 & 0.06\end{array}$

\$: HRFs are hemodynamic response function characteristics estimated with a fixed neuronal activity function. L-: left; R-: right; MFG: middle frontal gyrus; SMG: supramarginal gyrus; AG: angular gyrus; $H$ : height; $T$ : time to peak; $W$ : full width at half maximum of the HRF; A: additive genetic factors; C: common environmental factors; D: dominance genetic factors; E: unique environmental factors (E); -2LL: minus $2 \times 1$ loglikelihood; AIC: Akaike Information Criterion; $\Delta \chi 2$ : change in chi-square; $P$ : unadjusted $P$ values; aEb: a×10 .

Page 27 
Note that the $P$ values are conservative with the assumption of standard chi-square distribution. Given a null distribution of parameterized variance component being a 50/50 percent mixture of a $\chi^{2}(0)$ and $\chi^{2}(1)$ (Dominicus et al., 2006), the $P$ values should be half of the values reported in this table. 


\section{Table 4}

Statistical modeling of hemodynamic response function characteristics estimated with a flexible neuronal activity function§

\begin{tabular}{|c|c|c|c|c|c|c|c|c|c|c|c|c|c|c|c|c|c|c|c|c|}
\hline \multirow{3}{*}{ HRFs } & \multicolumn{10}{|c|}{ Model fit } & \multicolumn{10}{|c|}{ Model comparison } \\
\hline & \multicolumn{2}{|c|}{$\mathrm{ACE}$} & \multicolumn{2}{|c|}{$\mathrm{ADE}$} & \multicolumn{2}{|c|}{$\mathrm{CE}$} & \multicolumn{2}{|c|}{$\mathrm{AE}$} & \multicolumn{2}{|c|}{$\mathrm{E}$} & \multicolumn{2}{|c|}{ ACE vs. AE } & \multicolumn{2}{|c|}{ ACE vs. CE } & \multicolumn{2}{|c|}{ ADE vs. AE } & \multicolumn{2}{|c|}{ ADE vs. E } & \multicolumn{2}{|c|}{ AE vs. $\mathrm{E}$} \\
\hline & $-2 \mathrm{LL}$ & AIC & $-2 \mathrm{LL}$ & AIC & $-2 \mathrm{LL}$ & AIC & $-2 \mathrm{LL}$ & AIC & $-2 \mathrm{LL}$ & AIC & $\Delta \chi 2$ & $P$ & $\Delta \chi^{2}$ & $P$ & $\Delta \chi 2$ & $P$ & $\Delta \chi^{2}$ & $P$ & $\Delta \chi^{2}$ & $P$ \\
\hline L-MFG- $H$ & 752.68 & -159.32 & 752.64 & -159.36 & 755.40 & -158.60 & 752.68 & -161.32 & 768.47 & -147.53 & 0.00 & 1 & 2.72 & 0.10 & 0.04 & 0.84 & 15.83 & 3.64E-04 & 15.79 & 9 7.06E-05 \\
\hline L-SMG- $H$ & 498.34 & -177.66 & 496.80 & -179.20 & 501.77 & -176.23 & 498.34 & -179.66 & 505.80 & -174.19 & 0.00 & 1 & 3.43 & 0.06 & 1.53 & 0.21 & 9.00 & 0.01 & 7.47 & $6.29 \mathrm{E}-03$ \\
\hline L-AG- $H$ & 639.96 & -122.04 & 640.60 & -121.40 & 641.77 & -122.23 & 640.60 & -123.40 & 668.21 & -97.79 & 0.64 & 0.43 & 1.81 & 0.18 & 0 & 1 & 27.61 & 1.01E-06 & 27.61 & 1 1.48E-07 \\
\hline R-MFG- $H$ & 763.56 & -202.44 & 763.30 & -202.70 & 767.32 & -200.68 & 763.56 & -204.44 & 779.76 & -190.24 & 0.00 & 1 & 3.76 & 0.05 & 0.27 & 0.61 & 16.46 & 2.67E-04 & 16.19 & 9 5.72E-05 \\
\hline R-SMG- $H$ & 524.44 & -293.56 & 524.25 & -293.75 & 526.21 & -293.79 & 524.44 & -295.56 & 532.68 & -289.32 & 0.00 & 1 & 1.77 & 0.18 & 0.19 & 0.66 & 8.44 & 0.01 & 8.24 & $4.09 \mathrm{E}-03$ \\
\hline R-AG- $H$ & 693.44 & -174.56 & 692.18 & -175.82 & 696.41 & -173.59 & 693.44 & -176.56 & 700.67 & -171.33 & 0.00 & 1 & 2.96 & 0.08 & 1.26 & 0.26 & 8.50 & 0.01 & 7.23 & $7.16 \mathrm{E}-03$ \\
\hline L-MFG- $T$ & 1393.85 & 481.85 & 1393.85 & 481.85 & 1396.60 & 482.60 & 1393.85 & 479.85 & 1411.55 & 495.55 & 0.00 & 1 & 2.75 & 0.10 & $7.84 \mathrm{E}-0$ & 30.93 & 17.70 & 1.43E-04 & 17.69 & 9 2.60E-05 \\
\hline L-SMG- $T$ & 1173.10 & 497.10 & 1171.58 & 495.58 & 1174.98 & 496.98 & 1173.10 & 495.10 & 1176.49 & 496.49 & 0.00 & 1 & 1.88 & 0.17 & 1.52 & 0.22 & 4.91 & 0.09 & 3.39 & 0.06 \\
\hline
\end{tabular}




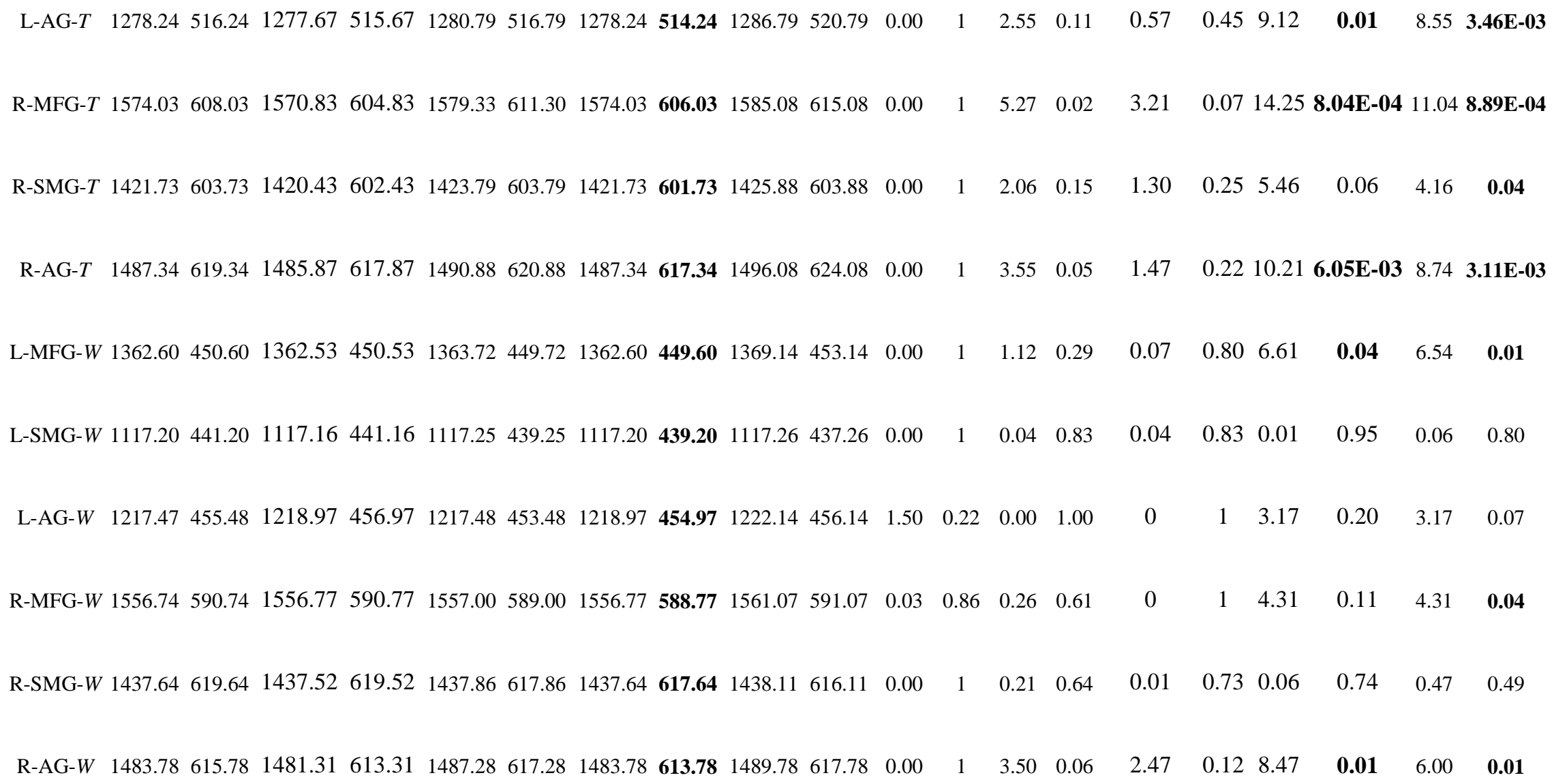

§: HRFs are hemodynamic response function characteristics estimated with a flexible neuronal activity function. L-: left; R-: right; MFG: middle frontal gyrus; SMG: supramarginal gyrus; AG: angular gyrus; $H$ : height; $T$ : time to peak; $W$ : full width at half maximum of the HRF; A: additive genetic factors; C: common environmental factors; D: dominance genetic factors; E: unique environmental factors (E); -2LL: minus 2×loglikelihood; AIC: Akaike information criterion; $\Delta \chi 2$ : change in chi-square; $P$ : unadjusted $P$ values; aEb: a $\times 10^{\mathrm{b}}$. 
Note that the $P$ values are conservative with the assumption of standard chi-square distribution. Given a null distribution of parameterized variance component being a 50/50 percent mixture of a $\chi^{2}(0)$ and $\chi^{2}(1)$ (Dominicus et al., 2006), the $P$ values should be half of the values reported in this table. 
Heritability estimates $(\%)^{\S}$

$H(95 \% \mathrm{CI}) \quad T(95 \% \mathrm{CI}) \quad W(95 \% \mathrm{CI})$

L-MFG $\quad \mathbf{4 3}^{* *}(\mathbf{1}, \mathbf{5 8}) \quad \mathbf{3 8}^{* *}(\mathbf{0}, \mathbf{5 3}) \quad 13(0,30)$

L-SMG $\quad \mathbf{3 6}^{*}(\mathbf{0}, \mathbf{5 6}) \quad \mathbf{4 4}^{*}(\mathbf{0}, \mathbf{6 5}) \quad 28(0,54)$

L-AG $\quad 36^{*}(0,55) \quad 34 *(0,53) \quad 43 *(6,61)$

R-MFG $36^{* *}(9,53) \quad 31 * *(0,48) \quad 31 * *(0,47)$

$\mathrm{R}-\mathrm{SMG} \quad \mathbf{3 7}^{* *} \mathbf{( 0 , 5 4 )} \quad \mathbf{5 1} 1^{* *}(\mathbf{2}, \mathbf{6 8}) \quad 21(0,44)$

R-AG $\quad 43 * *(14,60) \quad 33 *(\mathbf{0}, \mathbf{5 6}) \quad 31(0,57)$

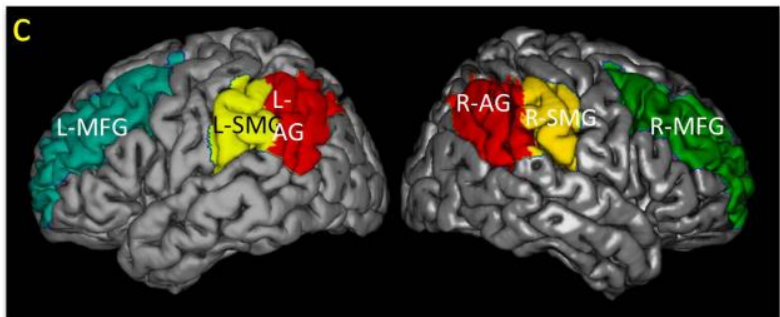

d

Heritability estimates $(\%)^{\S}$

$H(95 \% \mathrm{CI}) \quad T(95 \% \mathrm{CI}) \quad W(95 \% \mathrm{CI})$

L-MFG $\quad 40 * *(0,55) \quad 42 * *(0,57) \quad 23 *(0,39)$

L-SMG $\quad 47^{*}(\mathbf{0}, \mathbf{6 7}) \quad 24(0,47) \quad 3(0,27)$

L-AG $\quad 34 * *(\mathbf{0}, \mathbf{6 5}) \quad \mathbf{3 6}^{*}(\mathbf{0}, \mathbf{5 5}) \quad 0(0,35)$

R-MFG $\quad 42 * *(0,58) \quad 36 * *(8,54) \quad 14 *(0,35)$

R-SMG $\quad 31 *(\mathbf{0}, \mathbf{5 0}) \quad \mathbf{2 4} * \mathbf{( 0 , 4 5 )} 7(0,27)$

R-AG $\quad 33 *(0,53) \quad 37 *(3,56) \quad 26 *(0,45)$

\section{b}
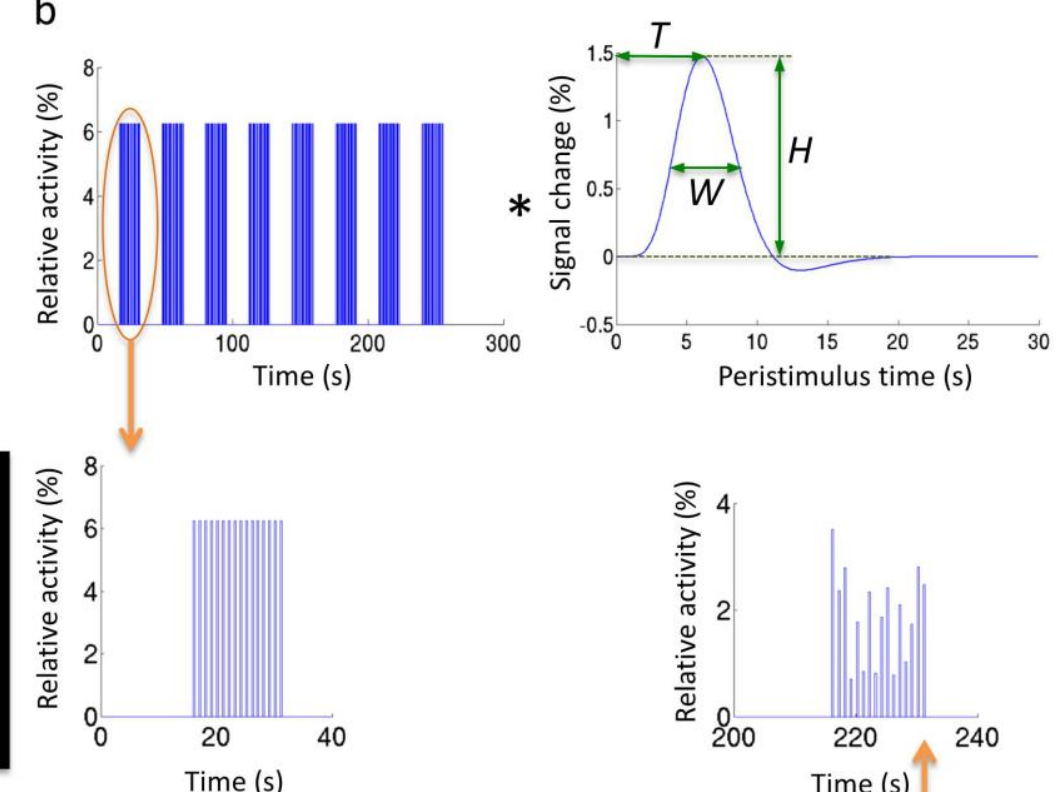

e
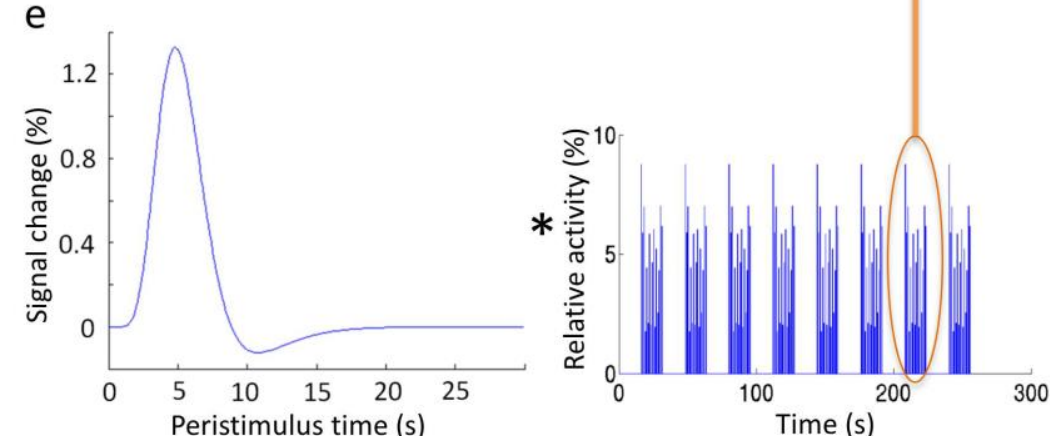

§: $H, T$, and $W$ : height, time to peak, and full width at the half maximum of the HRF; CI: confidence intervals; $* *$ : significantly decrease of goodness-of-fit with omission of genetic factors with Bonferroni correction $(P<0.0028)$ comparing $\mathrm{AE}$ with E modeling; *: moderately decrease of goodness-of -fit with omission of genetic factors $(P<0.05)$ comparing AE with E modeling.

Fig. 1

Page 32 


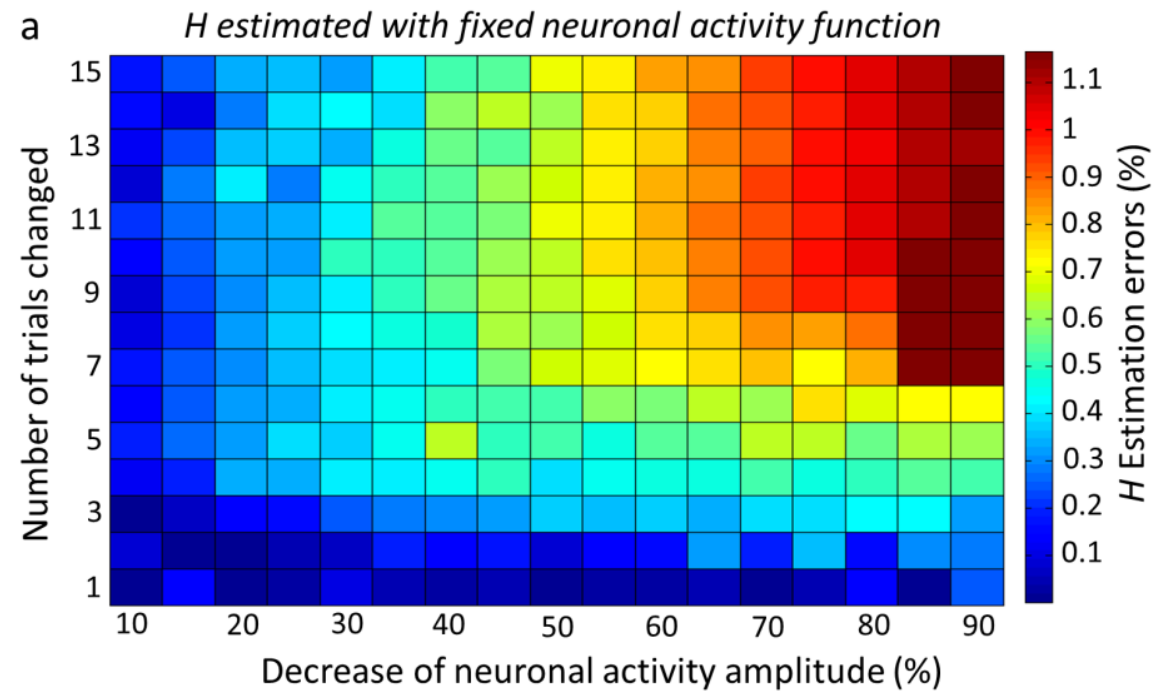

b Hestimated with flexible neuronal activity function
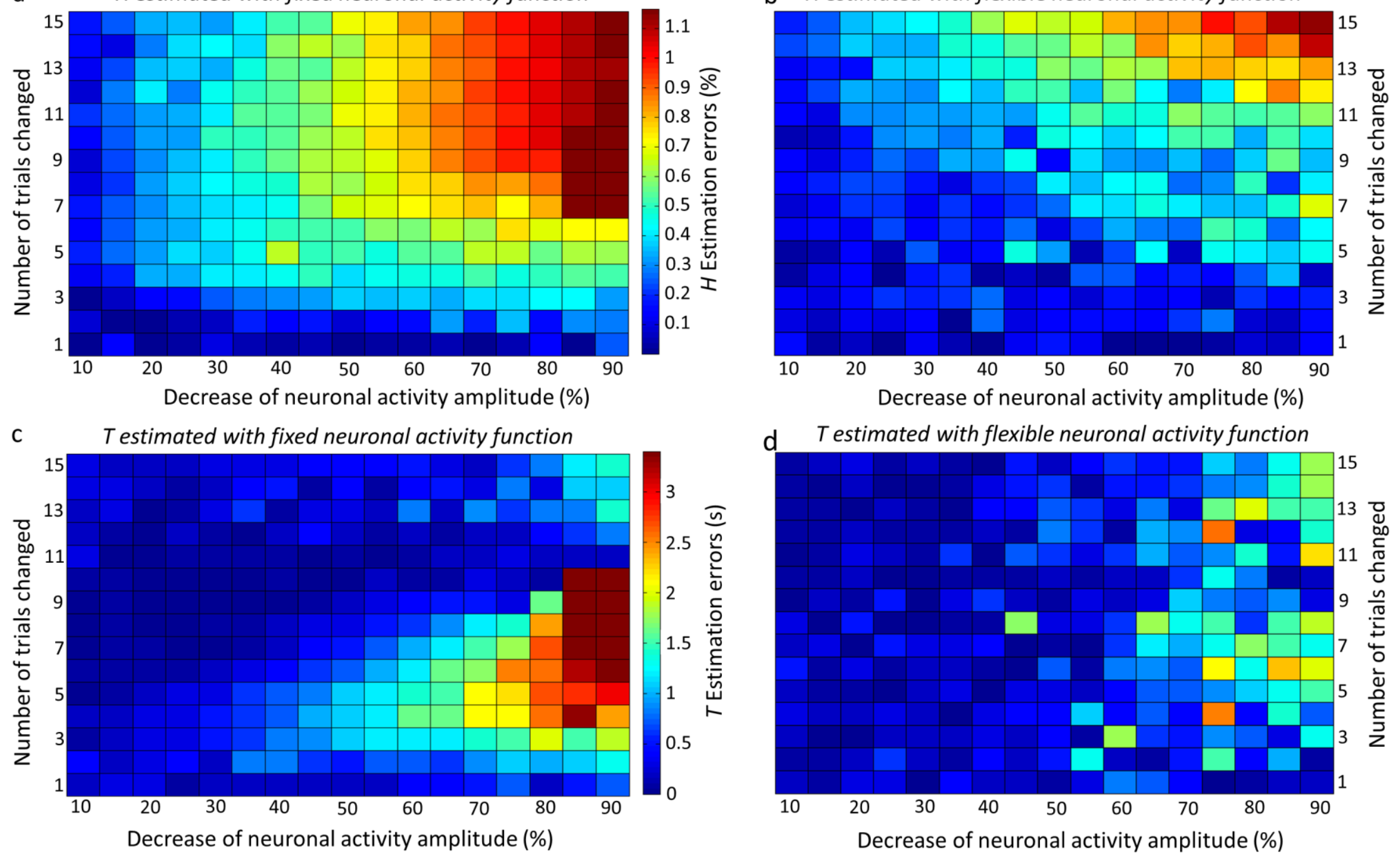

Fig. 2 


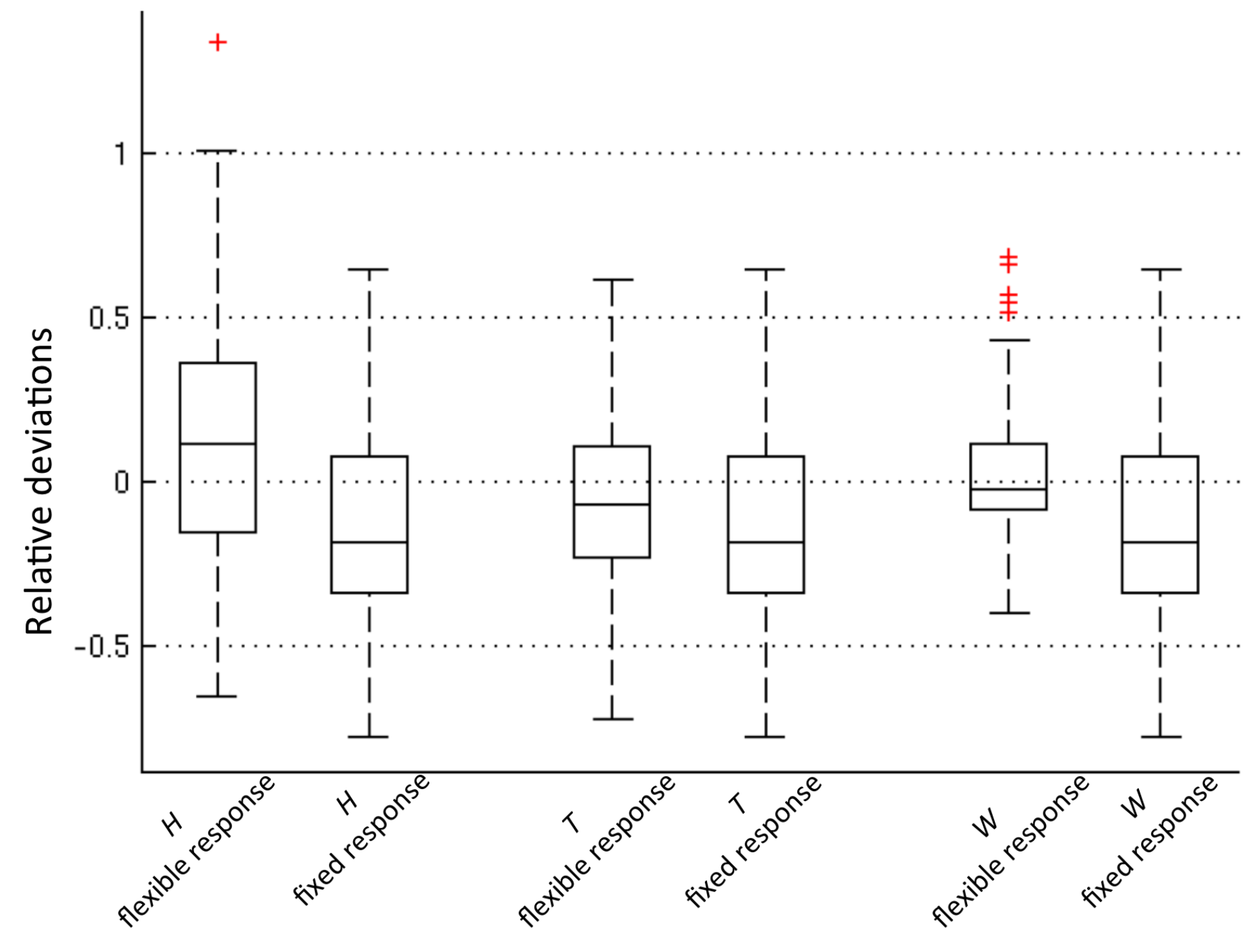

Fig. 3 
a

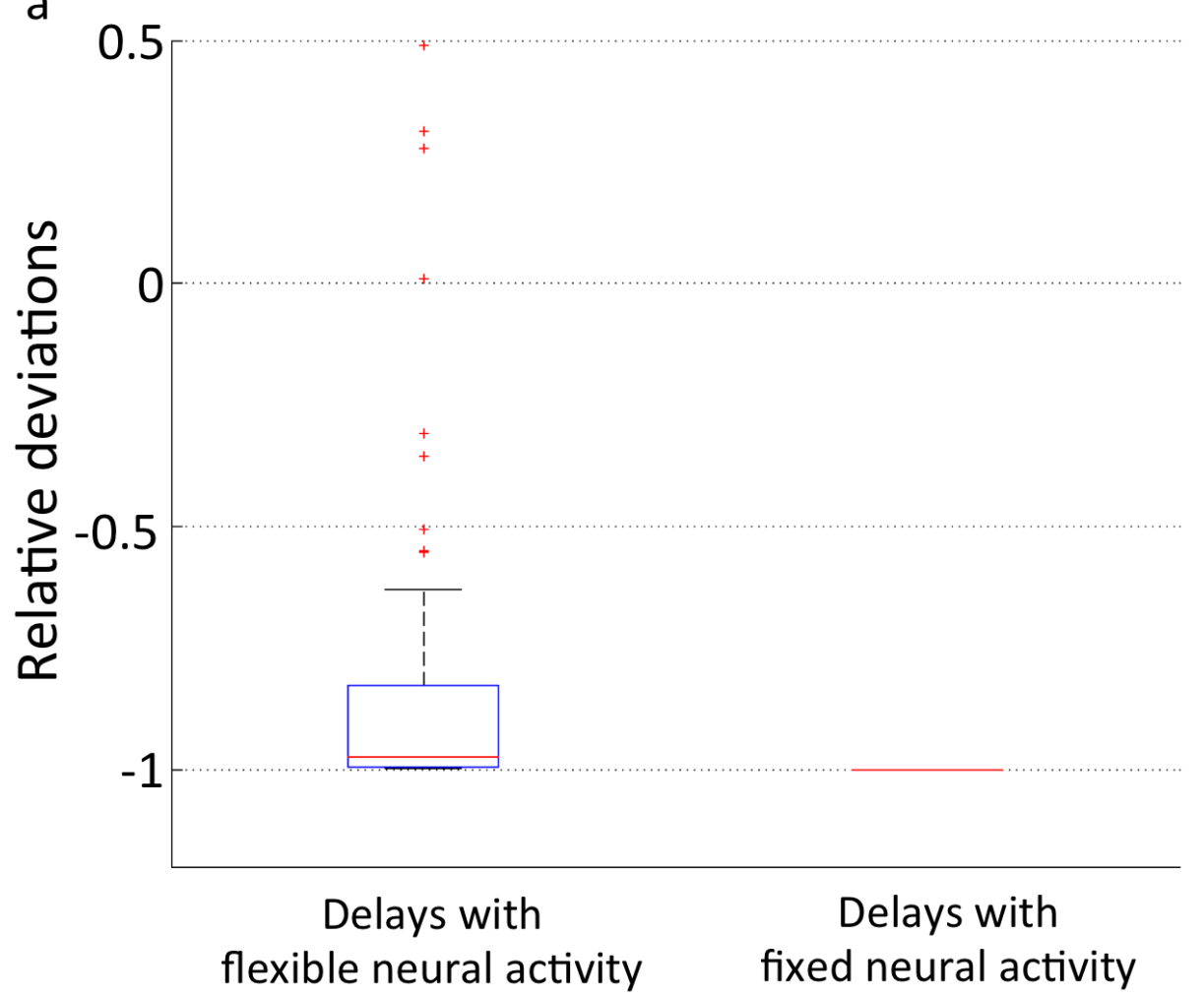

b

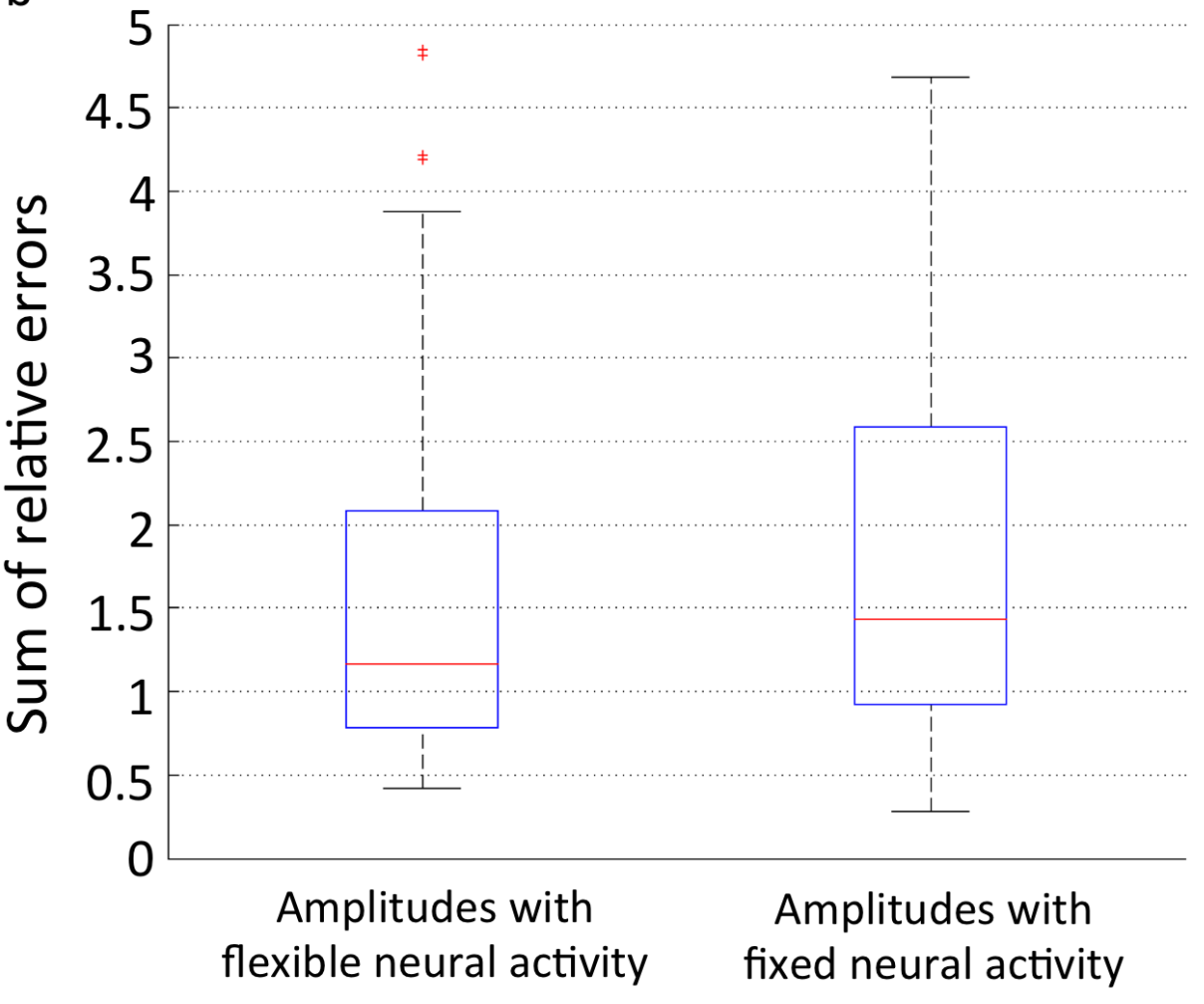

Fig. 4 


\section{Genes influence the amplitude and timing of brain hemodynamic responses}

\section{(Supplementary information)}

HRF sensitivity analysis. The factors affecting HRF shape were analyzed using the generalized Balloon Model \{Friston, 2000 \#67;Stephan, 2007 \#220\}. Neuronal activity triggers the vasodilatory signals that regulate changes in cerebral blood flow:

$$
\frac{d f(t)}{d(t)}=s \text { and } \frac{d s}{d t}=x(t+) \quad s \quad(f(t) 1)
$$

where $f(t)$ is the normalized blood flow, $s$ is the change of blood flow, $x(t)$ is vasodilatory signal triggered by neurovascular coupling, is the neurovascular coupling delay, is the vasodilatory signal decay and elimination of regional cerebral regulators such as nitric oxide, and is the autoregulatory feedback. The venous blood volume and change in deoxyhemoglobin concentration caused by the fluctuation in blood flow can be described as:

$$
\frac{d v}{d t}=f(t) \quad v(t)^{1 /} \quad \text { and }, \quad \frac{d q}{d t}=f(t) \frac{1 \quad\left(1 \quad E_{0}\right)^{1 / f(t)}}{E_{0}} v(t)^{1 /} \frac{q(t)}{v(t)}
$$

where $v(t)$ is the venous blood volume, is the transit time for blood to traverse the venous compartment of the vasculature, $\alpha$ is the vessel stiffness that determines the Windkessel properties of blood vessels, $q(t)$ is deoxyhemoglobin content and $E_{0}$ is the resting oxygen extraction fraction from capillary to tissue space. BOLD signal changes can be estimated from the venous volume and deoxyhemoglobin content:

$$
\frac{S}{S_{0}} \approx V_{0}\left[k_{1}(1 \quad q)+k_{2}\left(1 \frac{q}{v}\right)+k_{3}(1 \quad v)\right], k_{1}=4.3{ }_{0} E_{0} T E, k_{2}=r_{0} E_{0} T E, \text { and } k_{3}=1
$$

where $S$ is the BOLD signal, $V_{0}$ is the resting venous blood fraction $(0.03),{ }_{0}$ is the frequency offset at the outer surface of the magnetized vessel for fully deoxygenated blood determined by the magnetic field strength $\left(40.3 \mathrm{~s}^{-1}\right.$ for $\left.4 \mathrm{~T}\right), T E$ is the echo time for fMRI acquisition (0.03s), is the ratio of intra- and extravascular signals (0.4), and $r_{0}$ is the slope 
of the linear relationship between the intravascular relaxation rate and oxygen saturation (25 $\mathrm{s}^{-1}$ ), determined by magnetic field strength \{Friston, $2000 \# 67$;Stephan, $2007 \# 220$ \}. In theory, the vasodilatory signal, the neurovascular coupling delay, the vasodilatory signal decay, the autoregulatory feedback, the transit time, the vessel stiffness, the resting oxygen extraction fraction, and the ratio of intra- and extravascular signals can vary between individuals. In the sensitivity analysis, each parameter was systematically increased to $150 \%$ of its typical value, as estimated empirically in a previous study \{Friston, $2000 \# 67$, to determine its role in shaping the HRF.

The influences of physiological factors on HRF characteristics are illustrated in Fig. S1 and summarized in Table S1. $H$ is most strongly influenced by vasodilatory signalling and blood vessel stiffness, $T$ is most strongly influenced by neurovascular coupling delay and $W$ is most strongly affected by the venous transit time and the time constant of autoregulatory feedback.

Correlations between HRF characteristics and neuronal activations. Pearson correlations between HRF characteristics and neuronal activity changes were calculated using SPSS20 (IBM, New York). Statistical significance was determined using false discovery rate (FDR) corrected $P$ values (FDR-q < 0.05) with two tails (Storey, 2002).

The correlation between HRF characteristics and neuronal activity changes within the same location and subjects were summarized in Table S2.

Intra-class correlations of HRF characteristics between DZ pairs. For each HRF characteristic, the intra-class correlations (ICC) measuring absolute agreement in HRF characteristics within different types of DZ twin pairs $(\mathrm{DZM}=$ male $\mathrm{DZ}$ twin pairs, DZF = female DZ twin pairs, DZFM = DZ twin pairs with opposite genders) were determined with a two way random effects model using SPSS20 (IBM, New York). Tests of significant difference in ICC from zero was followed with Bonferroni correction $(P<0.0028)$ for multiple comparisons of $18 \mathrm{HRF}$ characteristics to determine statistical significance. The 
correlations and covariances of HRF characteristics within DZF, DZM, and DZFM are not significantly different.

Page 38 


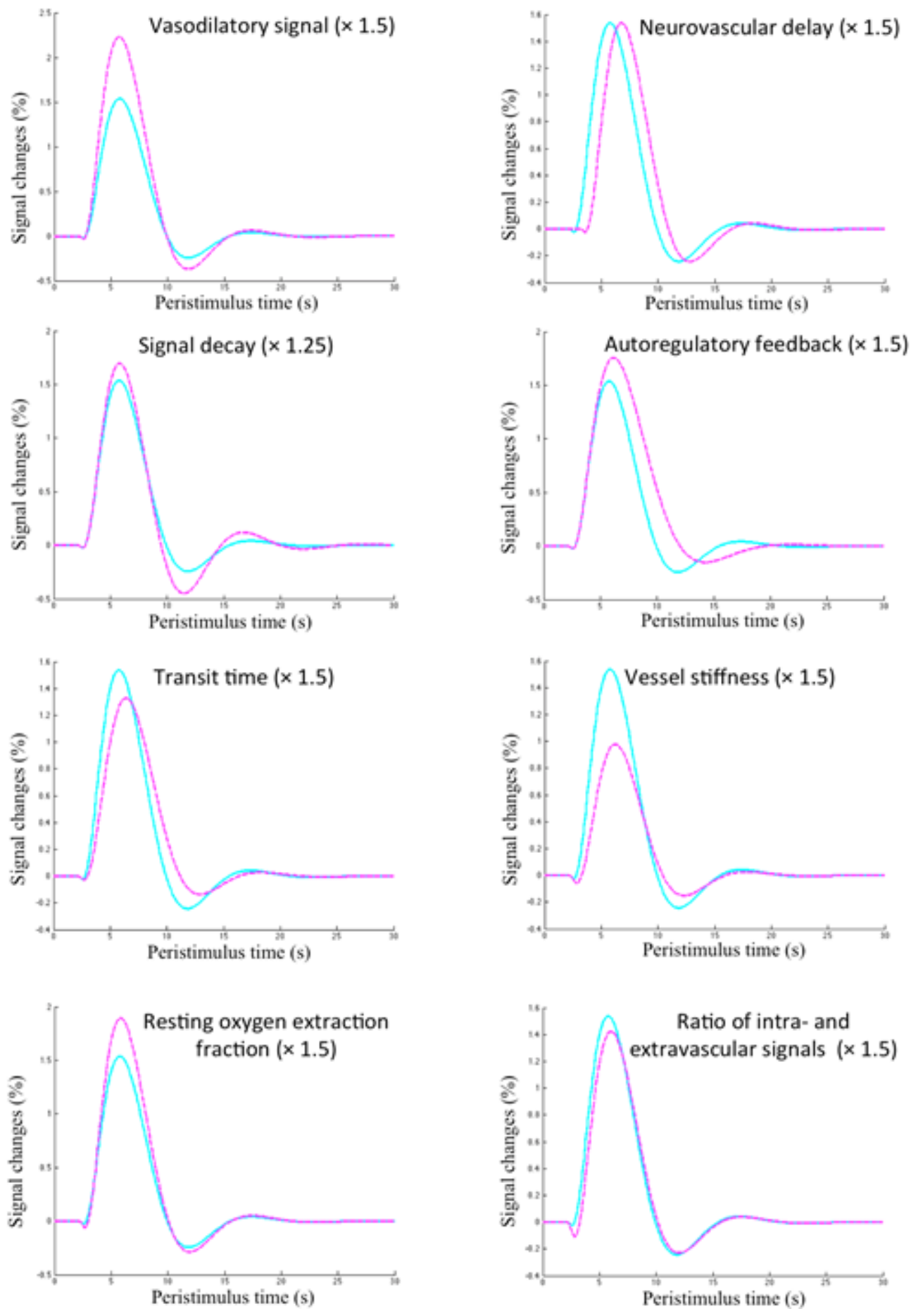

Fig. S1. Sensitivity analysis of the effect of varying parameters in the Balloon Model on the HRF. HRFs were generated using typical parameter values for the Balloon Model (cyan) or by increasing the specified parameter (magenta). All parameters were increased by $50 \%$ of their typical values except for signal decay, which was increased by $25 \%$ to stay within the range of plausible empirically observed HRFs. 


\section{Table S1}

Sensitivity of HRF characteristics to variables in Balloon $\mathrm{Model}^{\S}$

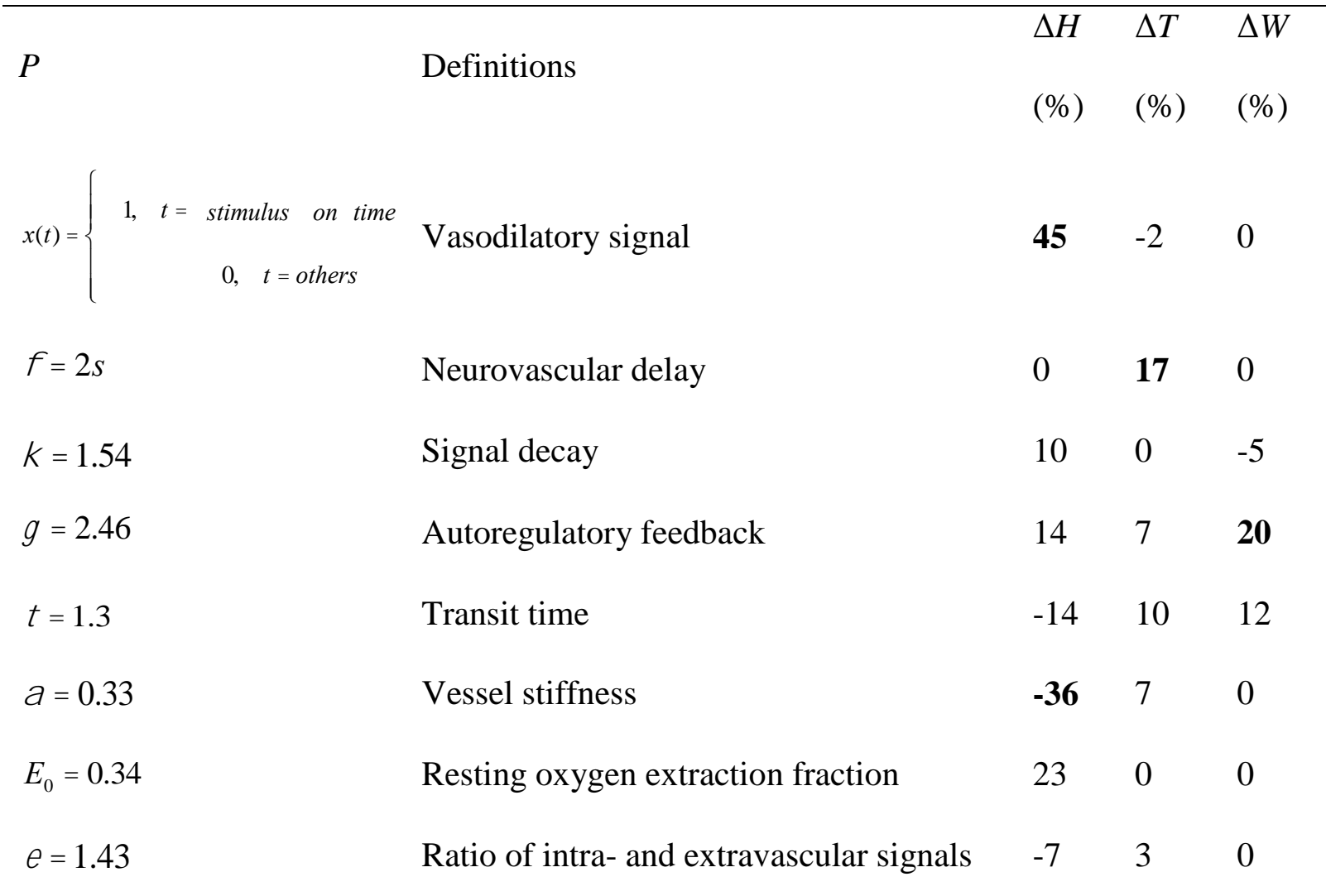

§: $P$ denotes the variable in Balloon Model. $\Delta H, \Delta T, \Delta W, \Delta O$ are the percentage change in HRF characteristic caused by increasing the specified variables by $50 \%$ except for , which was increased by $25 \%$.

Of the factors evaluated, vasodilatory signal and blood vessel stiffness had the most prominent influence on HRF $H$. HRF $T$ was mainly determined by neurovascular delay, while HRF $W$ was mainly affected by autoregulatory feedback and the transit time. 


\section{Table S2}

Correlations between HRF characteristics and neuronal activations for the same region of interest $(\mathrm{ROI})^{\S}$

\begin{tabular}{lcccc}
\hline ROI & $H \&$ Median & $H \&$ ApEn & $T \& \mathrm{D}$ & $W \&$ IQR \\
\hline L-MFG & $-0.091^{*}$ & $-0.101^{* *}$ & 0.026 & $0.191^{* *}$ \\
L-SMG & $-0.287^{* *}$ & $-0.133^{* *}$ & -0.055 & $0.135^{* *}$ \\
L-AG & $-0.125^{*}$ & $-0.056^{*}$ & 0.019 & $0.164 * *$ \\
R-MFG & $-0.241^{* *}$ & $-0.121^{* *}$ & -0.036 & $0.210^{* *}$ \\
R-SMG & $-0.311^{* *}$ & $-0.129^{* *}$ & -0.072 & $0.253^{* *}$ \\
R-AG & $-0.176^{* *}$ & $-0.128^{* *}$ & -0.01 & $0.198^{* *}$
\end{tabular}

$\S: H, T$ and $W$ are HRF characteristics of height, time to peak, and full width at half maximum; Median, ApEn, D and IQR are the median amplitude, the approximate entropy, delay, and the interquartile range of neuronal activity signal changes; No significant correlation was found if a relationship is not listed in the table, for an example, there is no significant correlation between HRF height and Delay of neuronal activity; L-: left; R-: right; MFG: middle frontal gyrus; SMG: supramarginal gyrus; AG: angular gyrus; **: significant correlations with FDR Q $<0.001 ; *$ : moderate correlations with FDR $\mathrm{Q}<0.01$.

Note that all measures here are relative signal changes. Therefore, the negative correlation between HRF $H$ and median of neuronal activity changes suggested that higher neuronal activity changes lower changes in HRF $H$. It does NOT suggest that higher neuronal activity with lower HRF $H$. 


\section{Table S3}

The intra-class correlations in HRF characteristics in DZ twin pairs ${ }^{\S}$

\begin{tabular}{|c|c|c|c|c|c|c|c|c|}
\hline \multirow{2}{*}{ HRFs } & \multicolumn{4}{|c|}{ Fixed neuronal activity functions } & \multicolumn{4}{|c|}{ Flexible neuronal activity functions } \\
\hline & $\mathrm{DZ}(95 \% \mathrm{CI})$ & DZF $(95 \%$ CI $)$ & DZM (95\% CI) & DZFM $(95 \% \mathrm{CI})$ & $\mathrm{DZ}(95 \% \mathrm{CI})$ & $\mathrm{DZF}(95 \% \mathrm{CI})$ & DZM (95\%CI) & DZFM $(95 \% \mathrm{CI})$ \\
\hline L-MFG- $H$ & $0.23(0,0.46)$ & $0.35(0,0.64)$ & $0.44(0,0.74)$ & $0.12(0,0.48)$ & $0.33 *(0.04,0.54)$ & $0.3(0,0.61)$ & $0.28(0,0.67)$ & $0.41 *(0,0.68)$ \\
\hline L-SMG- $H$ & $0.14(0,0.49)$ & $0.19(0,0.62)$ & $0.24(0,0.63)$ & $0.09(0,0.48)$ & $0.19(0,0.51)$ & $0(0,0.44)$ & $0.57(0,0.86)$ & $0.38(0,0.73)$ \\
\hline L-AG- $H$ & $0.2(0,0.47)$ & $0.11(0,0.56)$ & $0.33(0,0.69)$ & $0.1(0,0.54)$ & $0.49 *(0.19,0.68)$ & $0.68 *(0.27,0.86)$ & $0.52 *(0,0.8)$ & $0.34(0,0.7)$ \\
\hline R-MFG- $H$ & $0.03(0,0.31)$ & $0.07(0,0.47)$ & $0(0,0.47)$ & $0.13(0,0.5)$ & $0.36 *(0.11,0.54)$ & $0.39 *(0,0.64)$ & $0.28(0,0.67)$ & $0.41(0,0.66)$ \\
\hline R-SMG- $H$ & $0.07(0,0.4)$ & $0(0,0.43)$ & $0(0,0.53)$ & $0.27(0,0.55)$ & $0.17(0,0.46)$ & $0(0,0.41)$ & $0.19(0,0.68)$ & $0.33(0,0.67)$ \\
\hline R-AG- $H$ & $0.09(0,0.38)$ & $0(0,0.39)$ & $0(0,0.51)$ & $0.19(0,0.48)$ & $0.07(0,0.4)$ & $0(0,0.41)$ & $0.08(0,0.63)$ & $0.39(0,0.71)$ \\
\hline L-MFG- $T$ & $0.26(0,0.48)$ & $0.53(0.17,0.73)$ & $0.14(0,0.63)$ & $0.04(0,0.45)$ & $0.37 *(0.08,0.56)$ & $0.37 *(0,0.65)$ & $0.45(0,0.75)$ & $0.37(0,0.66)$ \\
\hline L-SMG- $T$ & $0.31(0,0.58)$ & $0.31(0,0.68)$ & $0.30(0,0.68)$ & $0.37(0,0.75)$ & $0.03(0,0.42)$ & $0(0,0.51)$ & $0.59(0,0.87)$ & $0.20(0,0.5)$ \\
\hline L-AG- $T$ & $0.08(0,0.39)$ & $0.24(0,0.62)$ & $0.22(0,0.68)$ & $0(0,0.36)$ & $0.18(0,0.49)$ & $0.41(0,0.75)$ & $0(0,0.48)$ & $0.09(0,0.58)$ \\
\hline
\end{tabular}




\begin{tabular}{|c|c|c|c|c|c|c|c|c|}
\hline R-MFG- $T$ & $0.01(0,0.3)$ & $0.19(0,0.53)$ & $0(0,0.16)$ & $0.12(0,0.48)$ & $0.15(0,0.39)$ & $0.22(0,0.54)$ & $0.45(0,0.74)$ & $0(0,0.38)$ \\
\hline R-SMG- $T$ & $0.28(0,0.53)$ & $0.41(0,0.71)$ & $0(0,0.56)$ & $0.36(0,0.68)$ & $0.03(0,0.36)$ & $0.1(0,0.53)$ & $0(0,0.33)$ & $0.27(0,0.64)$ \\
\hline R-AG- $T$ & $0.05(0,0.36)$ & $0.14(0,0.56)$ & $0(0,0.17)$ & $0.18(0,0.58)$ & $0.13(0,0.43)$ & $0.14(0,0.58)$ & $0(0,0.47)$ & $0.28(0,0.65)$ \\
\hline L-MFG- $W$ & $0.05(0,0.34)$ & $0.15(0,0.51)$ & $0(0,0.46)$ & $0.05(0,0.45)$ & $0.2(0,0.45)$ & $0.2(0,0.56)$ & $0.17(0,0.63)$ & $0.23(0,0.59)$ \\
\hline L-SMG- $W$ & $0.1(0,0.46)$ & $0(0,0.43)$ & $0.54(0.05,0.82)$ & $0.2(0,0.69)$ & $0.02(0,0.38)$ & $0.04(0,0.56)$ & $0(0,0.65)$ & $0(0,0.55)$ \\
\hline L-AG- $W$ & $0.2(0,0.47)$ & $0.1(0,0.55)$ & $0.24(0,0.68)$ & $0.26(0,0.62)$ & $0.4^{*}(0.04,0.63)$ & $0.19(0,0.66)$ & $0.63 *(0.14,0.85)$ & $0.44(0,0.73)$ \\
\hline R-MFG- $W$ & $0.15(0,0.39)$ & $0.37(0,0.63)$ & $0(0,0.45)$ & $0.09(0,0.47)$ & $0.2(0,0.43)$ & $0.28(0,0.58)$ & $0.17(0,0.63)$ & $0.23(0,0.59)$ \\
\hline R-SMG- $W$ & $0.17(0,0.46)$ & $0.17(0,0.60)$ & $0(0,0.55)$ & $0.33(0,0.66)$ & $0.03(0,0.43)$ & $0.15(0,0.57)$ & $0(0,0.52)$ & $0(0,0.48)$ \\
\hline $\mathrm{R}-\mathrm{AG}-W$ & $0.03(0,0.34)$ & $0(0,0.35)$ & $0(0,0.54)$ & $0.19(0,0.47)$ & $0.04(0,0.42)$ & $0.14(0,0.57)$ & $0(0,0.31)$ & $0(0,0.52)$ \\
\hline
\end{tabular}

§: Intra-class correlations (ICCs) were calculated using a two way random model and the type of absolute agreement. There was no significant effect of age and sex on HRF characteristics. HRFs: hemodynamic response function characteristics, DZ: all dizygotic twin pairs; DZF: dizygotic twin pairs with the same gender of female; DZM: dizygotic twin pairs with the same gender of male; DZFM: dizygotic twin pairs with opposite genders; L-: left; R-: right; MFG: middle frontal gyrus; SMG: supramarginal gyrus; AG: angular gyrus; Given that ICC cannot be negative, the negative ICC values rendered by SPSS were set to zeros; **: significant correlations with Bonferroni corrections $(P<0.0028)$; *: moderate correlations $(P<0.05)$. 